\title{
Biologie und Bewirtschaftung des Seesaiblings (Salvelinus alpinus L.) im Zugersee
}

\author{
Von Ch. Ruhlé \\ Institut für Gewässerschutz und Wassertechnologie an der ETH Zürich')
}

Manuskript eingegangen am 7. März 1977

\begin{abstract}
In recent years yields of the Alpine char fishery of Lake Zug have only been about $5 \%$ of traditional yields at the turn of the century.

Major results of a biological investigation of the species are presented. Management implications based on the biological study, that should reverse the trend of diminishing yields, are made.
\end{abstract}

\section{Einleitung}

Der Seesaibling des Zugersees ist ein Fisch mit Tradition. Er wird in einem Pfandrodel aus dem Jahre 1281, das eine wichtige Transaktion zwischen den Habsburgern und den Österreichern regelt, erstmals erwähnt [31], und wie in diesem Dokument wird er in verschiedenen anderen aus dem späteren Mittelalter als Zehnten oder Zins von verkauften, verschenkten oder verpfändeten Höfen aufgeführt. In den Jahren 1759 und 1788 muss sich der Zuger Rat mit ihm beschäftigen [30]; die beiden Ratsbeschlüsse regeln die gegenseitigen Rechte und Pflichten der Angel- und Netzfischer, die für die gemeinsame Ausübung des Rötelfanges obrigkeitlicher Vorschriften bedurften. Die Erneuerung dieser Vorschriften führte 1844 zur Gründung einer Fischereigesellschaft in Walchwil, die 100 Jahre später immer noch bestand.

Diese unvollständigen Angaben über die wenigstens marginale Beeinflussung der Zuger Lokalgeschichte durch den Seesaibling lassen verstehen, warum die Bevölkerung dort auch heute noch, obwohl jetzt vorwiegend in Verwaltung, Handel und Industrie tätig, ein stark ausgeprägtes «Rötel-Bewusstsein» hat. Dieses Bewusstsein hat sich nicht nur dadurch geäussert. dass diesem Fisch museale Würde verschafft wurde; es kommt auch im Erlass von Gesetzen zum Ausdruck, die die spezielle Förderung dieses «typischen» Fisches bezwecken. Und nicht zuletzt drückt es sich

1) Die Arbeit wurde an der Eidg. Anstalt für Wasserversorgung, Abwasserreinigung und Gewässerschutz (EAWAG), CH-8600 Dübendorf, ausgeführt. 
im Wunsch vorab der Zuger Regierung, aber auch vieler Geschäfte, Vereine und Einzelpersonen aus, jedes Jahr während der Rötelzeit wenigstens einmal die historische Delikatesse geniessen zu können.

Noch um die Jahrhundertwende und in den ersten Dekaden dieses Jahrhunderts fingen die Fischer des Zugersees viele Seesaiblinge. Der Zugersee wurde damals in der fischereilichen Seentypisierung [27, 63] einer der charakteristischsten Saiblingsseen genannt. Fangerträge von 90000 bis 100000 Seesaiblingen pro Jahr, die $85 \%$ des Gesamtfangertrages ausmachten [58], bestätigten die Richtigkeit der Zuordnung des Zugersees zu diesem Seetyp, der sich durch stenooligotrophe Eigenschaften, entsprechende Tiefe sowie ständig geringe Temperaturen und hohe Sauerstoffgehalte über das ganze Profil auszeichnet [27].

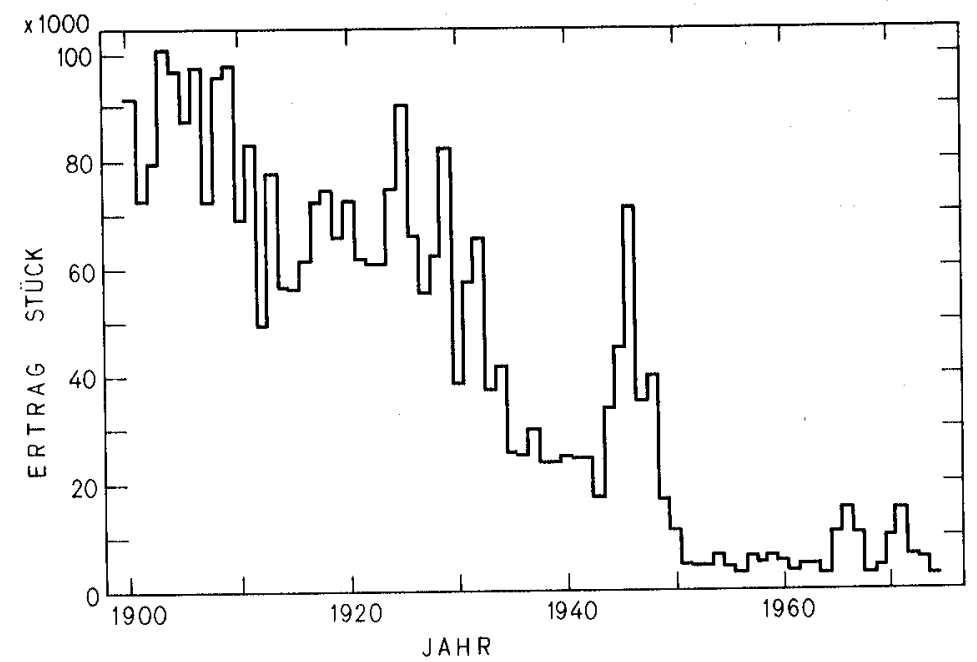

Abb. 1. Erträge der Seesaiblingsfischerei 1900-1974.

Figure 1. Yields (numbers of fish) of the char fishery 1900-1974.

Wie aus der Abb. 1 hervorgeht, hat sich die Situation seither stark verändert: im Durchschnitt der letzten 10 Jahre wurden lediglich 8400 Seesaiblinge gefangen; sie entsprechen ungefähr 2-3\% des gesamthaft erzielten Fanges.

Seit geraumer Zeit reichen nun diese Erträge nicht mehr aus, um die Nachfrage auch nur des einheimischen Marktes zu befriedigen, die auf ungefähr 20000 Stück geschätzt wird [46]. Dies (und dies vielmehr als der aus der Diskrepanz zwischen Angebot und Nachfrage resultierende hohe Preis) sowie die Überzeugung der wichtigsten Abnehmer, der Wirte und Hoteliers, dass der einheimische Fisch aus qualitativen Gründen nicht einfach durch Seesaiblinge aus anderen Seen ersetzt werden könne, hat schon zu verschiedenen Versuchen geführt, die Seesaiblingsfischerei und -bewirtschaftung zu sanieren. Die gesteckten Ziele wurden jedoch nicht erreicht. Es entstand darum das Bedürfnis, im grösseren Rahmen als bisher die Biologie des Seesaiblings im Zugersee zu studieren und Bewirtschaftungsmassnah- 
men auszuarbeiten, deren Anwendung eine weitere Abnahme der Erträge verhindern oder sogar eine Zunahme ermöglichen sollte.

\section{Beschreibung des Zugersees}

\subsection{Geographie, Geologie, Morphometrie und Hydrologie}

Der Zugersee ist ein Voralpensee (Mitte der Längsachse: $47^{\circ} 04^{\prime}$ n. B., $8^{\circ} 29^{\prime}$ ö.L.), der zwischen der Rigi (ca. $1800 \mathrm{~m}$ ü. M.), dem Rossberg (1600 m), dem Zugerberg $(1000 \mathrm{~m})$ und Ausläufern des Rooterberges $(850 \mathrm{~m})$ liegt (Abb. 2). Die nähere Umgebung des Sees besteht vorwiegend aus Molasse-Mergel und Molasse-Nagelfluh.

Zwei Halbinseln ragen vom Westen her in den See; die grössere, der «Kiemen» (Chiemen) teilt den See in zwei morphologisch unterschiedliche Teile, in den Obersee (Südteil) und in den Untersee (Nordteil). Die tiefste Stelle des Sees liegt im zuflussärmeren, abflussentfernten Obersee. Der Zugersee liegt im Hoheitsgebiet dreier Kantone: Zug (ca. 65\% der Oberfläche), Schwyz (ca. 25\%) und Luzern (ca. $10 \%)$.

Die wichtigsten morphometrischen und hydrologischen Daten des Zugersees sind in Tabelle 1 zusammengefasst.

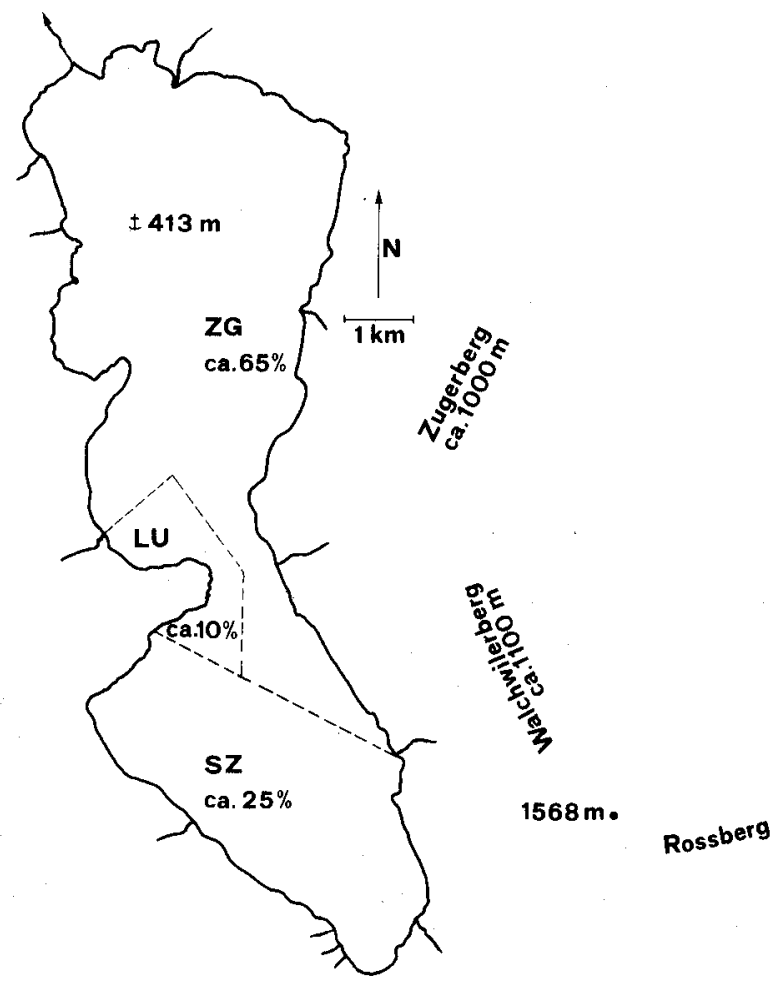

Rigi $\bullet 1797 m$

Abb. 2. Zugersee. Figure 2. Lake Zug. 


\subsection{Limnologie}

Der Zugersee ist erst relativ spät Gegenstand limnologischer Untersuchungen geworden (Ausnahme: Brutschy [12]) und wird erst seit einigen Jahren periodisch überwacht. Seine Entwicklungsgeschichte konnte indessen gut von Züllig [66] anhand von Bohrkernen aus dem Sediment nachverfolgt werden. Die von ihm bestimmten Gehalte an Gesamt-P, Gesamt-N, Gesamt-S, Sulfid-Schwefel, organischem Kohlenstoff sowie freier und geformter Kieselsäure weisen bis in die Zeit um die Jahrhundertwende eine gewisse Konstanz auf. Die erwähnten Gehalte steigen in den Sedimenthorizonten für die Zeit nach 1900 steil an. In dieser Zeit (1907-1909) stellte Brutschy [12] auch die ersten Massenentwicklungen gewisser Plankter fest. Über die Entwicklung des Sees seither kann der Vergleich von Transparenz (Tab. 2) und Färbung (Tab.3) einen wenn auch oberflächlichen Eindruck vermitteln. Die Angaben stammen von Brutschy [12] und Zeder [65].

Sein heutiger, eutropher Zustand ist aus dem Vergleich einiger Grenzwertkriterien für die Zuordnung zum eutrophen Seetyp und zu den entsprechenden Ist-Werten des Zugersees (Tab. 4) ersichtlich.

\subsection{Fischereiverhältnisse}

Wie eingangs erwähnt wurde, galt der Zugersee um die Jahrhundertwende noch als typischer Saiblingssee. Zwischen dem Bau des Beckens und dem Eutrophierungsgrad bestand eine «natürliche Bindung» [27].

Der Bruch dieser Bindung durch äussere Einflüsse manifestiert sich in einer Änderung der Artenzusammensetzung (Abb. 3) und in einer Erhöhung des Gesamtfangertrages (Abb.4). Beide Erscheinungen sind typische Folgeerscheinungen der Eutrophierung, wie wir sie mit Ausnahme von einigen weniger beeinflussten Voralpen- und Alpenseen von den meisten Schweizer Seen her kennen.

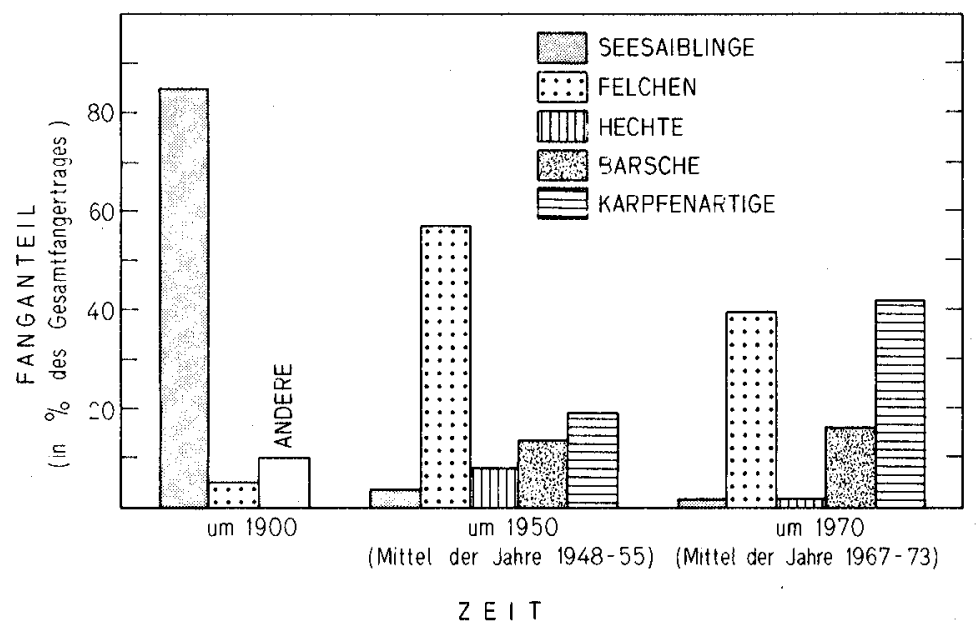

Abb. 3. Änderungen in der Artenzusammensetzung der Fänge, 1900-1970.

Figure 3. Changes of the species composition of the catches, 1900-1970, expressed as percentage of total catch. 


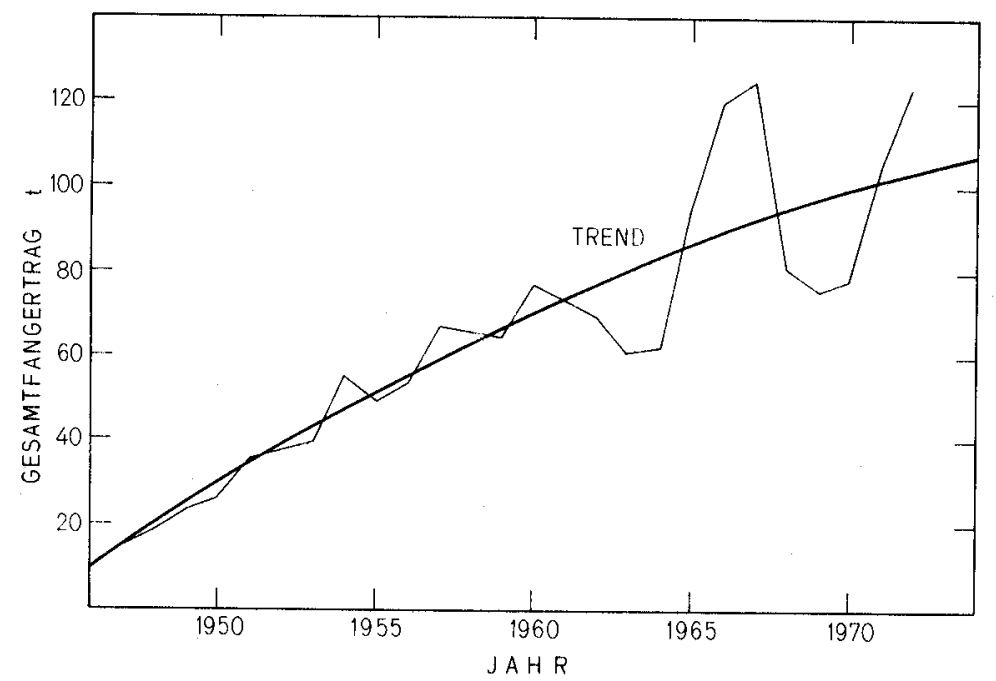

Abb. 4. Änderungen im Gesamtfangertrag der Zugerseefischerei, 1946-1973.

Figure 4. Changes of the total yield of the Lake Zug fishery, 1946-1973.

Die Veränderung der Artenzusammensetzung zugunsten der wenig verlangten Cypriniden äussert sich in einer Abnahme des durchschnittlichen pro Einheit erzielten Verkaufserlöses und, gleicher fischereilicher Aufwand für den Fang der verschiedenen Arten vorausgesetzt, in einer Reduktion des aufwandbezogenen Verdienstes der Berufsfischer. Am Zugersee waren im Jahre 1975 noch deren 15 beschäftigt. 9 davon waren hauptberuflich oder vorwiegend in der Fischerei tätig; 6 gingen der gewerbsmässigen Fischerei nur im Nebenberuf nach. Der langfristig auch am Zugersee feststellbaren Abnahme der Anzahl Berufsfischer steht eine Zunahme der Bedeutung der Sportfischerei gegenüber. Im Jahre 1973/74 lösten 800 Personen ein Sportfischerpatent. Dem Fiskus strömten aus dem Verkauf dieser Bewilligungen Fr.27000.- oder 1,7mal mehr als für den Erlös aus den Berufsfischerpatenten zu. Im Durchschnitt der Jahre 1967 bis 1972 waren die Sportfischer mit etwa $7 \%$ am gesamthaft erzielten Fangertrag beteiligt.

\section{Allgemeine Charakterisierung des Seesaiblings (Salvelinus alpinus L.)}

Die Seesaiblinge nehmen in der Systematik folgende Stellung ein:

Ordnung: Clupeiformes $=$ Heringsfische

Unterordnung: Salmonoidei $=$ Lachsartige

Familie: Salmonidae $=$ Lachse

Unterfamilie: Salmoninae = kleinschuppige Lachse

Gattung: Salvelinus $=$ Saiblinge

Art/Rassenkreis: Salvelinus alpinus $=$ See- bzw. Wandersaibling

Die Saiblinge des Rassenkreises Salvelinus alpinus (Rassenkreis nach Rensch [43]: «ein Komplex geographischer Rassen, die sich unmittelbar auseinander entwickelt 
haben, geographisch einander vertreten und von denen jeweils die benachbarten miteinander unbegrenzt fruchtbar sind $"$ ) sind Zirkumpolarfische. Sie kommen in der Neuen und Alten Welt sowic auf den arktischen Inseln vor [35]. Sie leben anadrom (Wandersaiblinge) oder stationär (Seesaiblinge). Nach Nikolsky [36] sollen jedoch auch Populationen existieren, bei denen das Verhalten hinsichtlich Wanderung nicht eindeutig ist. Er erwähnt zudem Seesaiblinge, die erst in jüngster Zeit wegen künstlicher oder natürlicher Barrieren aus anadromen Saiblingen hervorgegangen sind.

Der unterschiedlichen Lebensweise messen verschiedene Autoren ungleiche systematische Bedeutung bei $[8,10,34,35]$, was sich in einer uneinheitlichen Namengebung äussert (Salvelinus alpinus, Salvelinus salvelinus, Salvelinus alpinus salvelinus). Gerade die heute noch mögliche Überführung von anadromen Saiblingen in stationär lebende Saiblinge weist aber darauf hin, dass Seesaiblinge wie Wandersaiblinge Vertreter eines Rassenkomplexes Salvelinus alpinus sind. In älteren Publikationen wird der Seesaibling oft der Gattung Salmo zugeordnet. Im Gegensatz zu diesen hat er jedoch [10]:

1. viel kleinere Schuppen,

2. entsprechend mehr Schuppen entlang der Seitenlinie (mindestens 195; Vertreter der Gattung Salmo 120-130),

3. ein kurzes, anders bezahntes Pflugscharbein,

4. nicht mehr als 50 Pylorusanhänge ( $S$. trutta bis 60, S. salar bis 74).

Die Saiblinge des Rassenkreises Salvelinus alpinus kommen auf dem europäischen Kontinent in Zentraleuropa, auf den Britischen Inseln und in Skandinavien (inkl. Island) vor. Die heutige südliche Verbreitungsgrenze der Wandersaiblinge verläuft ungefähr entlang des 64. Breitengrades (Südküste Islands) [23].

In Zentraleuropa bewohnt der Seesaibling die kalten, sauerstoffreichen Seen der Alpen nördlich ihres Zentralkammes [50]. Sie sollen dort bis fast $2800 \mathrm{~m}$ ü. M. vorkommen [42]. Auf der Alpensüdseite fehlen sie. Gegen Ende des letzten Jahrhunderts wurden sie jedoch aus dem Zugersee in den Luganersee [42, 57] und von dort im Jahre 1910 in den Langensee eingeführt [26].

In der Alpennordschweiz scheint der Seesaibling früher in den meisten Seen vertreten gewesen zu sein. Wieweit die Populationen aber autochthon waren, lässt sich heute nicht mehr feststellen. Es bestehen Anhaltspunkte dafür, dass er zumindest im Thuner- und Brienzersee eingebürgert wurde [62]. Er fehlte wahrscheinlich im Murten-, Pfäffiker-, Greifen-, Lungern-, Sarner- und Hallwilersee.

Die ursprüngliche Verbreitung des Fisches in Europa und die südliche Verbreitungsgrenze der Wandersaiblinge lassen erkennen, dass es sich bei den Wander- wie Seesaiblingen um kaltstenotherme Fische handelt. Als Salmoniden haben sie ein hohes Sauerstoffbedürfnis.

\section{Die Biologie des Seesaiblings im Zugersee}

\subsection{Die Ernährung}

Der Fang der adulten, freilebenden Seesaiblinge findet im Zugersee zur Hauptsache während der Laichzeit auf den Laichgründen statt. Die Magen der nahezu oder 
ganz laichreifen Fische waren meistens leer.

Die wenigen untersuchten Seesaiblinge, die nicht aus der Laichzeit stammten (Beifänge der Felchen- und Barschfischerei), wiesen in ihren Mageninhalten folgende Futterorganismen auf (Tab. 5).

Die auffallende Dominanz von Corethra, die vorab in eutrophen Gewässern vorkommt [17] und die in einigen der untersuchten Mageninhalte als einziger Organismus in sehr grosser Zahl (bis 3000) vorhanden war, sowie die durchaus hohen Konditionsindizes der untersuchten Fische weisen darauf hin, dass die Seesaiblinge des Zugersees das durch die Eutrophierung des Sees veränderte Nahrungsangebot auszunützen vermögen.

Junge, unter künstlichen Bedingungen aufgezogene Seesaiblinge weisen eine Bevorzugung von Cyclops und Bythotrephes auf. Diese Organismen können im Magen selbst dann noch nachgewiesen werden, wenn sie im entsprechenden Futterangebot kaum mehr feststellbar sind. Daphnia wird nur dann in grösserer Anzahl aufgenommen, wenn das Futter nichts oder fast nichts anderes enthält. Diaptomus wird ungefähr in der Proportion seiner Vertretung im Futter aufgenommen.

\subsection{Das Wachstum}

Die den Wachstumsberechnungen zugrunde liegenden Daten wurden nach Standardverfahren erhoben:

- Schuppenentnahme sowie Längen- und Gewichtsmessungen an vorwiegend lebenden (anästhesierten) Fischen.

- Schuppeninterpretation auf einem Projektionsmikroskop.

- Rückberechnung aufgrund einer Segestraleschen Eichkurve SchuppengrösseFischlänge, an die die individuelle Schuppe durch einen Korrekturfaktor angepasst wurde. Derjenige Bereich der Eichkurve, der für kleine Fische gilt, wurde aufgrund von Daten ermittelt, die von künstlich aufgezogenen Vorsömmerlingen, Sömmerlingen und Jährlingen stammen.

Für die Wachstumsberechnungen wurden Altersbestimmungen an insgesamt 389 Fischen vorgenommen; 223 waren Weibchen, 166 Männchen. Altersbestimmungen an Seesaiblingsschuppen sind nach verschiedenen Autoren [4, 25, 37, 42] schwierig durchzuführen. Die Entnahme innerer Organe war indessen wegen des hohen Marktwertes der Fische und der Gewohnheit, sie lebend zu verkaufen, nicht möglich. Die Altersbestimmungen wurden an markierten Fischen bekannten Alters kontrolliert. Die Kontrollen ergaben keine Hinweise für Fehlinterpretationen.

Die Wachstumskurve für das Längenwachstum, die nach von Bertalanffy [9] die allgemeine Form

$$
y=A\left(1-e^{-x k}\right)
$$

hat und sich in bezug auf Längen in

$$
1_{t_{i}}=L_{\max }\left(1-e^{-k\left(t_{i}-t_{0}\right)}\right)
$$


umschreiben lässt, wurde mit Hilfe eines von Allen [3] stammenden Programms berechnet. Die Berechnung der Gewichtswachstumskurve mit der Form

$$
g_{t_{i}}=a \cdot L_{\max }^{v}\left(1-e^{k\left(t_{0}-t_{i}\right)}\right)^{v}
$$

basiert auf obengenannter Formel für das Längenwachstum und der LängenGewichte-Beziehung

$$
\mathrm{g}=\mathrm{a} \cdot \mathrm{L}^{\mathrm{v}} .
$$

In den Abb. 5, 6 und 7 ist der Verlauf des Längenwachstums, die Beziehung zwischen den Längen und den Gewichten sowie der Verlauf des Gewichtswachstums (inkl. Gewichtszuwachs) wiedergegeben. In der Tabelle 6 sind die Kurvenwerte für die Längenwachstumskurven enthalten.

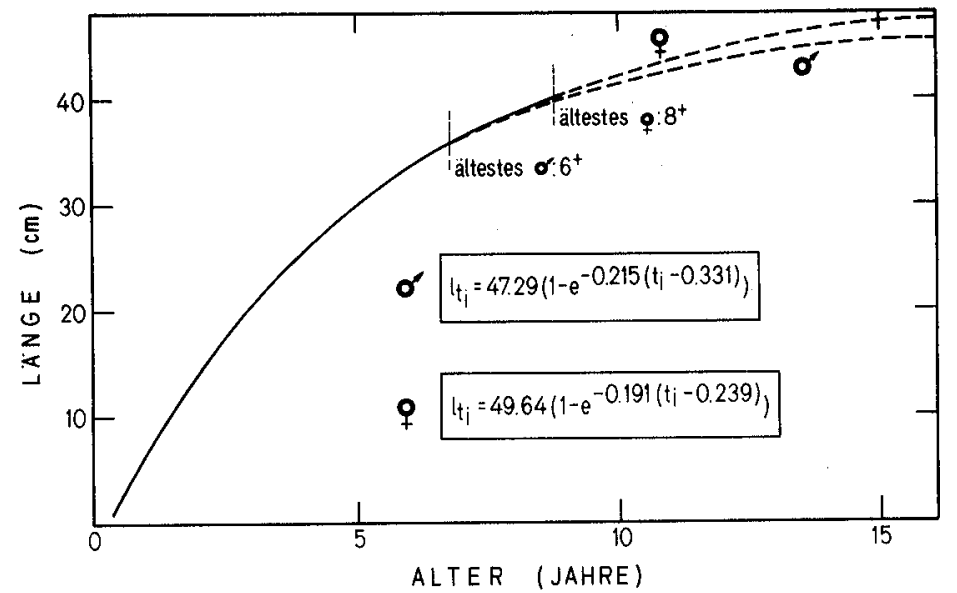

Abb. 5. Verlauf des Längenwachstums.

Figure 5. Age-length relationship.

Bemerkenswert sind folgende Ergebnisse:

- Das Längenwachstum der Männchen und Weibchen unterscheidet sich gesamthaft gesehen kaum. Allfällige Unterschiede sind im Extrapolationsbereich feststellbar.

- Weibchen sind bei gleicher Länge schwerer als Männchen.

- Demzufolge unterscheidet sich das Gewichtswachstum beider Geschlechter; das Gewichtszuwachsmaximum wird von den Männchen ungefähr ein Jahr früher als von den Weibchen erreicht.

(Im Zusammenhang mit der Frage, wieweit der Einsatz ganz junger Fische wegen der Ausnützung in tieferen Stufen der Trophiepyramide produzierter Biomasse sinnvoll ist, sei auf die geringen Zuwachsleistungen in diesem Alter 


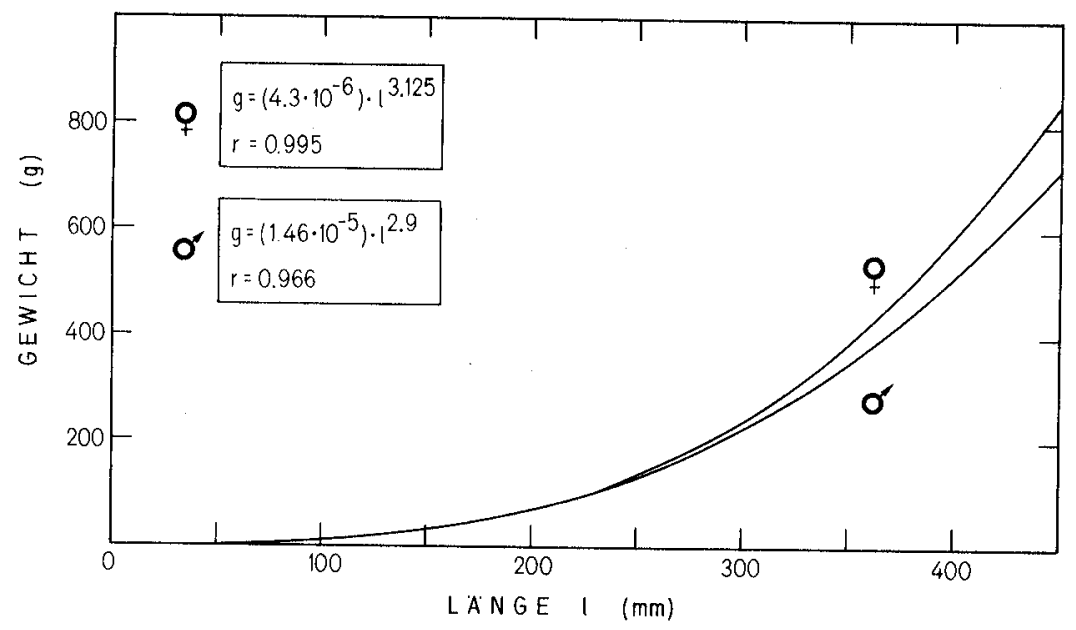

Abb. 6. Beziehung zwischen Länge und Gewicht.

Figure 6. Length-weight relationship.

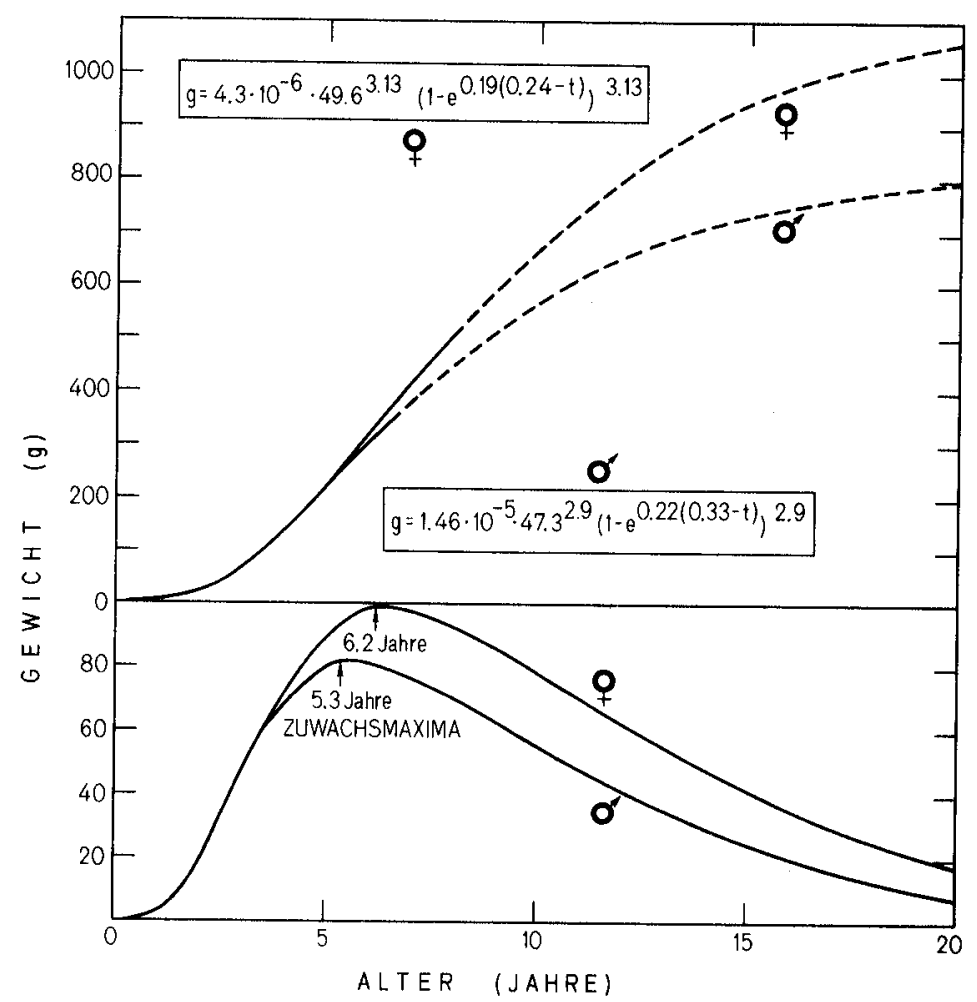

Abb. 7. Verlauf des Gewichtswachstums; Gewichtszuwachs.

Figure 7. Age-weight relationship and annual weight increases. 
und auf die daraus ableitbare geringe Ausnützung dieser Biomasse hingewiesen.)

Die Berechnung des Längenwachstumsverlaufes von 4 Gruppen von Fischen, die aufgrund ihrer rückberechneten Länge am Ende des ersten Lebensjahres ein sehr gutes (Länge im Alter 1: >79 mm), gutes $(69-79 \mathrm{~mm}$ ), mittleres (59$68 \mathrm{~mm}$ ) bzw. schlechtes Startwachstum $(<59 \mathrm{~mm})$ aufwiesen, zeigt, dass diese unterschiedliche Wüchsigkeit im späteren Leben beibehalten wird.

\subsection{Die Fortpflanzung}

Die Fortpflanzung bei den Saiblingen des Salvelinus-alpinus-Komplexes ist entsprechend ihres vielgestaltigen Verbreitungsgebietes und ihrer unterschiedlichen Lebensweisen uneinheitlich.

Deutliche Unterschiede bestehen hinsichtlich:

- Länge der Laichwanderung, vorab als Folge unterschiedlicher Aufenthaltsgebiete der Wander- und Seesaiblinge während der Ernährungsphase. Innerhalb der Salmoninae gilt die Gattung Salvelinus als diejenige mit der am wenigsten ausgeprägten Anadromie (eine nichtwandernde Art: Salvelinus namaycush) [29]; trotzdem kann auch bei den meisten süsswasserstabilen Saiblingspopulationen ein mehr oder weniger klar ausgebildetes Wanderverhalten (See-Fluss, Fluss-Fluss, See-See) festgestellt werden [49].

- Auswahl der Laichgewässer (stehend oder fliessend).

- Alter beim Eintritt der Geschlechtsreife: $1^{+}[37]$ bis $11^{+}$oder $12^{+}[2,25]$.

- Entwicklungsdauer der Eier (solche anadromer Saiblinge offensichtlich kürzer als die süsswasserstabiler).

Als Gemeinsamkeit kann die Eiablage und Eientwicklung unter günstigen respiratorischen Bedingungen, meistens in kiesigen Gründen (lithophile Fische [36]) aufgeführt werden. Saiblinge scheinen zudem grundsätzlich pluripar zu sein.

Die Untersuchungen, die über die Fortpflanzung der Seesaiblinge des Zugersees durchgeführt wurden, betreffen den Eintritt der Geschlechtsreife, die Entwicklung zur Laichreife (inkl. geschlechtsgebundener Unterschied in dieser Entwicklung: Protandrie), die Fruchtbarkeit und die Entwicklungsdauer der Eier sowie die Fortpflanzung unter natürlichen Bedingungen im See:

Wie schon im Kapitel über die Ernährung dargelegt wurde, werden die Seesaiblinge des Zugersees fast ausschliesslich während der Laichzeit und auf den Laichgründen gefangen. Dementsprechend schwierig war es, die Gonadenentwicklung im jahrzeitlichen Ablauf zu verfolgen. Fest steht lediglich, dass die Rückbildung der Keimdrüsen im Mai mehrheitlich abgeschlossen ist (Reifegrad II nach Kiseljowitsch) und dass in der Zeit zwischen Juni und Oktober das relative Gonadengewicht ständig bis zur vollen Reife zunimmt. Die Population wird als laichreif erachtet, wenn $50 \%$ der gefangenen Weibchen laichreif sind (Stadium V). Im langjährigen Durchschnitt ( 65 Jahre) ist dies am 15. November ( \pm 4 Tage) der Fall.

Ende Oktober erscheinen die ersten Seesaiblinge, die sich während der Ernährungsphase vorwiegend im Untersee, im Litoral und Pelagial aufgehalten hatten, auf den Laichgründen des Obersees (heute noch deren 25 in Tiefen zwischen 25 und $80 \mathrm{~m}$ ). 


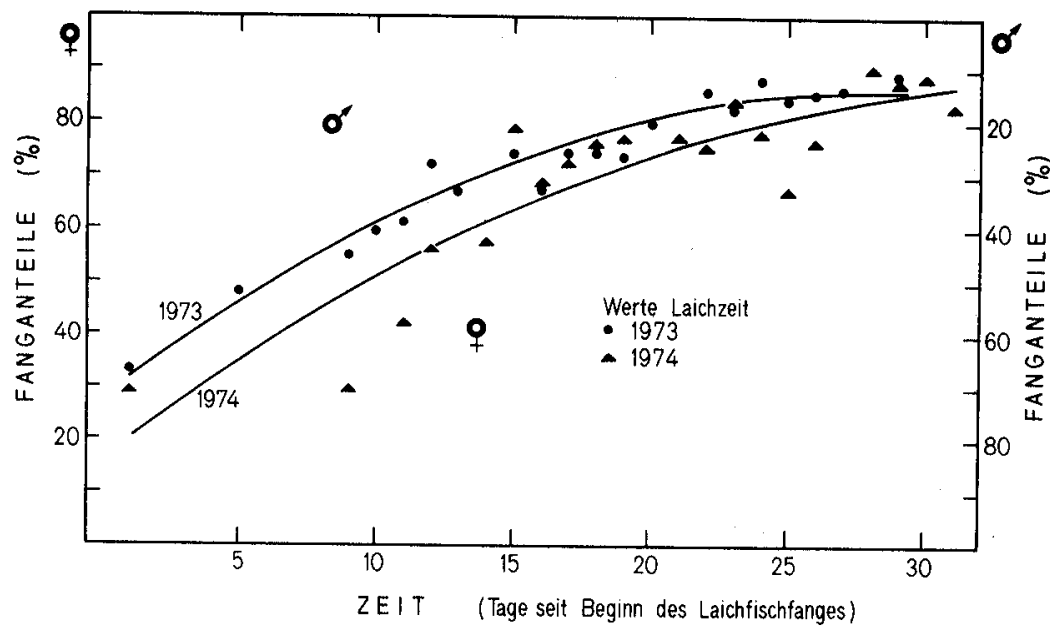

Abb. 8. Geschlechtsgebundener Unterschied beim Eintreffen auf den Laichgründen.

Figure 8 . Sex-specific difference in time of reaching the spawning grounds.

Wie aus der Abb. 8 hervorgeht, besteht hinsichtlich des Eintretens der Laichreife und dem Eintreffen der Fische auf den Laichgründen zwischen Männchen und Weibchen ein beachtlicher Unterschied. Diese Erscheinung (Protandrie) ist zum Teil wenigstens darauf zurückzuführen, dass für die Ovulation und das Laichverhalten des Weibchens ein mehrtägiges, vorangehendes Laichgebaren der Männchen Bedingung ist [64]. Der zeitliche Unterschied in der Reifung der Männchen und Weibchen kann zu weitreichenden negativen Folgen für die künstliche Besamung führen.

Unter natürlichen Bedingungen braucht diese Verschiebung und das Geschlechtsverhältnis, das mit etwa 65\% Weibchen (1973: 71,2\%, 1974: 63,5\%) wesentlich vom natürlichen Geschlechtsverhältnis von ungefähr 1:1 [36,59] abweicht, nicht die gleichen Konsequenzen zu haben. Es scheint vielmehr so zu sein, dass die Männchen unter ungestörten Bedingungen längere Zeit auf den Laichgründen bleiben [20] und sich offensichtlich mit mehreren Weibchen "paaren» [21]. Der frühere Eintritt der Laichreife bei den Männchen ist demnach mit einer länger dauernden Laichreife gekoppelt, die diejenige der Weibchen überlappt, eine Erscheinung, die selbstverständlich in einer während der Laichzeit intensiv befischten Population nicht zu erkennen ist.

Eine Analyse der Altersklassenzusammensetzung des Fanges aus der ordentlichen Laichfischerei der Jahre 1971 bis 1973 ergab die in Tabelle 7 (oberer Teil) zusammengefassten Resultate.

Diese Resultate müssen a priori wegen der Selektivität der gesetzlich vorgeschriebenen Netze mit mindestens $30 \mathrm{~mm}$ Maschenweite in Frage gestellt werden. Die Ergebnisse der Kontrollfänge mit feineren Netzen sind aus dem unteren Tabellenteil ersichtlich. Sie lassen (unter Berücksichtigung dessen, was eben über das Geschlechtsverhältnis gesagt wurde) folgende Schlüsse hinsichtlich des Alters der Seesaiblinge beim Eintritt der Geschlechtsreife zu: Eintritt der Geschlechtsreife im 
Alter $1^{+}$: mit grosser Wahrscheinlichkeit ausgeschlossen, $2^{+}$: möglich, besonders bei Männchen, $3^{+}$: wahrscheinlich, $4^{+}$: möglich, besonders bei Weibchen.

Suworow [59] definiert die Fruchtbarkeit von Fischen mit der Anzahl abgegebener Eier. Diese Definition steht nicht im Einklang mit anderen, die ihr die Zahl reifender oder reifer Eier zugrunde legen [6]. Die Suworowsche Definition wurde besonders deshalb angewandt, weil es nicht möglich war, Fische in genügender Zahl zu töten. Die Fruchtbarkeit der Seesaiblinge des Zugersees wurde an 87 Weibchen bestimmt. Je 40 Eier von 55 dieser Weibchen wurden gemessen. Die Ergebnisse der Fruchtbarkeitsbestimmungen sind aus Abb. 9 zu entnehmen.

Erwartungsgemäss steigt die Eizahl mit zunehmender Fischlänge. Die nichtlineare Beziehung hat die allgemeine Form $F=a \cdot L^{b}$, wobei $F=$ Fruchtbarkeit, Fertilität, Eizahl bedeutet. Der Exponent $b$ dürfte im allgemeinen einen Wert um 2 haben (Kisselewitch, zit. nach Suworow [59]). Bagenal [7] hat Werte gefunden, die zwischen 2,34 und 5,28 liegen. Der niedrige von mir berechnete Exponent von 1,4 ist in erster Linie darauf zurückzuführen, dass zu wenig grosse und alte Weibchen berücksichtigt werden konnten. Aus der linearen Beziehung zwischen Körpergewicht und Eizahl lässt sich die relative Fruchtbarkeit (n Eier $/ \mathrm{kg}$ Weibchen) bestimmen. Für die Seesaiblinge des Zugersees beträgt sie 7782,83. Der Unterschied zwischen den Eizahlen der Weibchen verschiedenen Alters ist statistisch gesichert.

Die Eimessungen ergaben, dass sich die Eigrösse bei zunehmender Fischgrösse nicht ändert. Es konnte damit auch nicht der Frage nachgegangen werden, ob grösseren Eiern grössere und kräftigere Nachkommen als kleineren Eiern entstammen [7, 13]. Unter künstlichen Erbrütungsbedingungen schlüpfen die Seesaiblinge des Zugersees nach etwa 380 Tagesgraden; der Unterschied zwischen warm und kalt erbrüteten ist gering: $12^{\circ} \mathrm{C}: 384,3{ }^{\circ} \mathrm{C}: 381$ Tagesgrade. Nach Bigelow [10] dauert die Eientwicklung (anadromer ?) Saiblinge 270-315 Tagesgrade.

Bemerkenswert ist, dass sich die unterschiedlichen Wassertemperaturen uneinheitlich auf die einzelnen Entwicklungsabschnitte auswirken: die Entwicklung bis zum

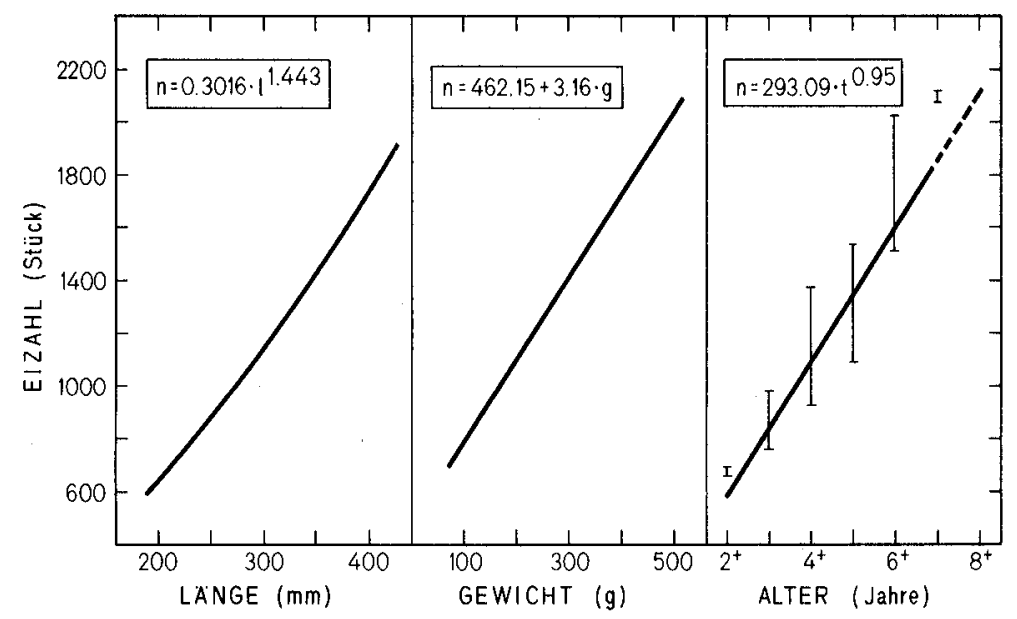

Abb. 9. Abhängigkeit der Eizahl von der Fischlänge, des Fischgewichtes und des Fischalters. Figure 9. Relationship between egg number and total length, weight, and age of fish, respectively. 
Augenpunktstadium dauert im $3{ }^{\circ} \mathrm{C}$ warmen Wasser 2,8 mal länger als im $12^{\circ} \mathrm{C}$ warmen; für die Entwicklung vom Augenpunktstadium bis zur Larve (Schlüpfen) beträgt dieser Faktor 6,5, vom Schlüpfzeitpunkt bis zur Dottersackresorption 1,9.

Von entscheidender Bedeutung für die Seesaiblingsbewirtschaftung ist die Frage, ob und wieweit sich die Fische im Zugersee noch natürlich fortpflanzen können. Die Beantwortung dieser Frage in bezug auf eine der beiden Funktionen der Laichgründe, nämlich auf die der Stimulation der Fische, sich für die Laichablage auf ihnen einzufinden, war relativ einfach. Schon die grossen Erträge, die während der Laichzeit auf einigen der heute noch befischten Laichgründe erzielt werden, liefern die Gewissheit, dass wenigstens diese Laichgründe in ihrer Kornzusammensetzung den Bedürfnissen der laichenden Fische entsprechen. Nach Fabricius und Gustafson [21] ziehen 92 von 100 Seesaiblingen Laichgründe aus feinem Kies (6), grobem Kies (53), kopfsteinpflastergrossen Steinen (23) oder aus in Sand eingebetteten Steinen (11) für die Laichablage anderen, feiner strukturierten Gründen vor. Die Bevorzugung grobkiesiger Böden konnte auch im Zugersee festgestellt werden. Sie kommt in einer recht deutlichen Abhängigkeit der Anzahl Fische, die sich auf den Laichgründen einfinden (ausgedrückt durch die Anzahl pro fischereilichen Aufwand unter vergleichbaren Bedingungen gefangener Fische), vom Zustand (photographisch ermittelt: siehe Abb. 10, 11) der Laichgründe zum Ausdruck (Abb. 12).

Einige Schwierigkeiten bot die Beantwortung der Frage, ob auf natürliche Weise Seesaiblingseier in die Laichgründe gelangen und, vor allem, ob sich diese unter den im eutrophen Zugersee herrschenden Bedingungen entwickeln können. Die Mindestzahl der im See abgelaichten Eier wurde für die Jahre 1973 und 1974 auf 200000 bzw. 70000 geschätzt. Diese Annahme basiert auf der Untersuchung von 5127 gefangenen Weibchen (1973: 3385, 1974: 1742), von denen 44,16 bzw. 31,1\% «leer» waren. Dass wenigstens ein Drittel dieser «leeren» Weibchen unter natürlichen Bedingungen zum Laichen kamen und die Eier nicht einfach durch das

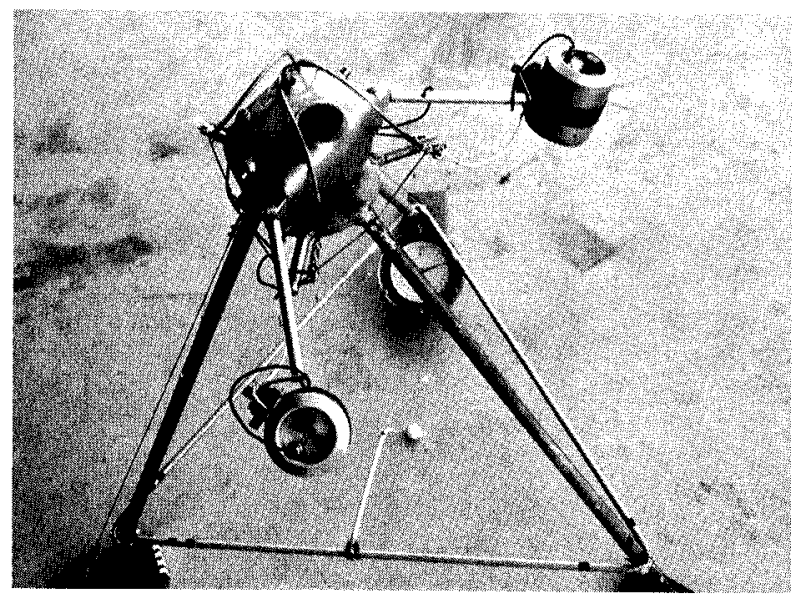

Abb. 10. Unterwasserkamera mit Blitzgeräten auf Dreibeinstativ mit Eisenfüssen, inversem Lot und Manometer.

Figure 10. Underwater camera, mounted on a tripod with iron feet, perpendicular and manometer. 
Fischen (Trommelsucht) oder durch unsachgemässe Behandlung verloren hatten, geht aus der Tatsache hervor, dass die Regressionsgerade zwischen dem (jeden Tag erhobenen) Anteil verlaichter Weibchen und der Zeit seit Beginn der Laichfischerei in beiden Jahren einen praktisch identischen Verlauf und ein vergleichbares Bestimmtheitsmass hat; die Höhe dieses Anteils ist somit von variablen äusseren Einflüssen (Fischerei) nicht wesentlich beeinflusst.

Die direkte Überprüfung der Funktionstüchtigkeit der Laichgründe in bezug auf die Erbrütung der abgelegten Eier wurde auf drei verschiedene Arten versucht:

- Anwendung einer Schlittendredge [15].

- Ausheben von Laichgründen (wie z. B. von Roth und Geiger [44] praktiziert); diese Arbeit wurde im See von Tauchern ausgeführt.

- Anwendung eines Kleinschleppnetzes [54] vor dem Einsatz künstlich erbrüteter und aufgezogener Jungfische zum Nachweis unter natürlichen Bedingungen entwickelter Seesaiblinge.

Die Ergebnisse aller drei Verfahren liessen keine eindeutigen Schlüsse hinsichtlich der Funktionstüchtigkeit der Seesaiblingslaichgründe als Entwicklungsmedium für Eier zu. Erfolgreicher war der Einsatz von Vibert-ähnlichen Käfigen [60], in die befruchtete Eier gelegt waren und die im See über den Laichgründen versenkt wurden. In einem dieser versenkten Käfige konnte wenigstens ein Erbrütungserfolg von $1 \%$ nachgewiesen werden. In allen anderen Käfigen waren alle Eier abgestorben und mit einer schwarzen Schicht von offensichtlich unter schlechten $\mathrm{O}_{2}$-Bedingungen abgebautem organischem Sediment bedeckt. Um die Gewissheit zu erlangen, dass die Abgänge nicht methodisch bedingt waren, wurden verschiedene Kontrollversuche durchgeführt (Erbrütung des gleichen Laichmaterials im Brutsieb, Anwendung des Vibert-ähnlichen Käfigs unter günstigen respiratorischen Bedingungen im Trog, im Freiwasser des Zugersees, im Walensee). Die Resultate dieser Kontrollen

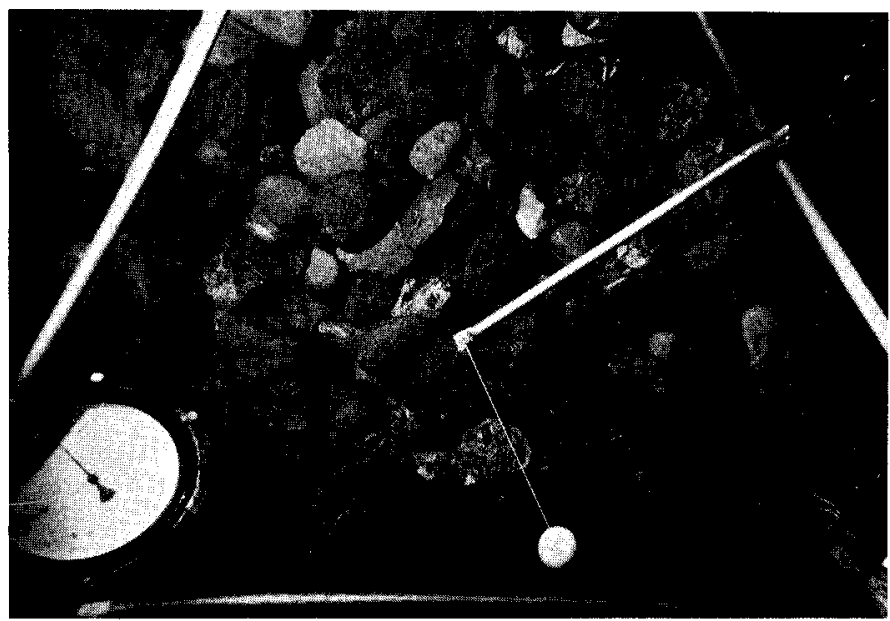

Abb. 11. Photographische Aufnahme eines grobkiesigen Laichgrundes; die Lage des inversen Lotes weist auf einen extrem steilen (ca. $63^{\circ}$ ) Laichgrund hin.

Figure 11. Photograph of a coarse gravel spawning ground. The position of the perpendicular line with the ball indicates an extremely steep spawning ground. 


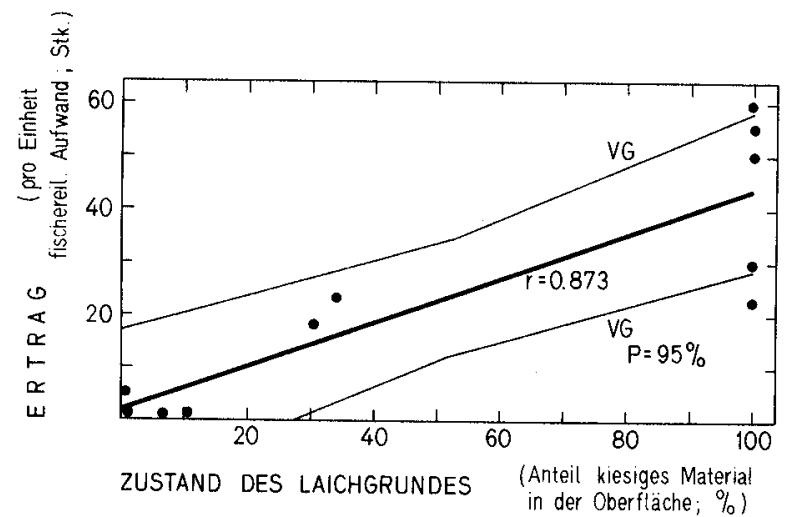

Abb. 12. Abhängigkeit des Attraktionsvermögens der Laichgründe (ausgedrückt durch den pro fischereilichen Aufwand erzielten Ertrag) vom Laichgrundzustand (Anteil kiesiges Material in der Oberfläche).

Figure 12. Relationship between fish attraction capacity of the spawning grounds (expressed as catch per fishing effort) and the condition of the spawning grounds (percentage of coarse gravel on the ground surface).

bestätigten indessen die Ergebnisse des Versuchs, die eigentlich nicht erstaunen können, wenn die besonderen $\mathrm{O}_{2}$-Verhältnisse im Wasser über Grund und (für Eier, die, wenn sie natürlich abgelaicht werden, im Seegrund liegen) im Interstitialwasser sowie die $\mathrm{O}_{2}$-Bedürfnisse von Salmonideneiern berücksichtigt werden:

- Nach Angaben von Aldridge et al. [1] nimmt der Sauerstoffbedarf von Salmonideneiern mit fortschreitender Entwicklung zu. Am Ende der Eientwicklung beträgt der Critical oxygen content $\left(\mathrm{O}_{2}\right.$-Gehalt, bei dem eine ungestörte Entwicklung noch sicher ist) $z$. B. für $O$. keta $7 \mathrm{mg} / 1$.

- Hayes und Macauley [28] haben die $\mathrm{O}_{2}$-Zehrung des Sedimentes aus dem darüberliegenden Wasser in einem als meso bis eutroph einstufbaren See mit $11 \mathrm{mg} / \mathrm{dm}^{2}$ Tag bestimmt.

- Nach Provoni [39] ist die $\mathrm{O}_{2}$-Aufnahme aus dem über Grund liegenden Wasser nicht so sehr von den trophischen Bedingungen als vom $\mathrm{O}_{2}$-Gehalt dieses Wassers (und der Temperatur) abhängig. Bei einem Gehalt von $5 \mathrm{mg} / 1$ beträgt nach ihm die Zehrung etwa $20 \mathrm{mg} / \mathrm{m}^{2} \mathrm{~h}=4,8 \mathrm{mg} / \mathrm{dm}^{2} \mathrm{Tag}$.

- Die durchschnittliche mittlere Tiefe der Seesaiblingslaichgründe im Zugersee beträgt $50 \mathrm{~m}$. In dieser Tiefe sind $\mathrm{O}_{2}$-Gehalte von 5 bis $7 \mathrm{mg} / 1$ während der Laichzeit und der darauffolgenden Wochen die Regel. Recht häufig wurden aber schon niedrigere Gehalte (3-4 mg/l) gemessen.

Diese Hinweise allein erklären bereits die geringen Bruterfolge bei den in Käfigen versenkten Eiern. Für die Entwicklung der in die Laichgründe eingebetteten natürlich abgelaichten Eier muss noch der $\mathrm{O}_{2}$-Gehalt im und die fraktionsabhängige Geschwindigkeit der Erneuerung des Interstitialwassers mit in die Betrachtung einbezogen werden:

- Nach Eltringham [16] (basiert auf [11]) ist bei einem Feinsandanteil von nur etwa $15 \%$ mit einem auf $10 \%\left(\mathrm{O}_{2}\right.$-gesättigtes Wasser über Grund $\left.=100 \%\right)$ reduzierten Sauerstoffgehalt im Interstitialwasser zu rechnen. 
- Nach Glass und Poldoski [24] verringert sich der $\mathrm{O}_{2}$-Gehalt im Interstitialwasser der oberen $2,5 \mathrm{~cm}$ dicken Sedimentschicht des Lake Superior von $12,7 \mathrm{mg} / 1$ auf $5.3 \mathrm{mg} / \mathrm{l}$. Das Verhältnis $\mathrm{O}_{2}$-Gehalt Interstitialwasser: $\mathrm{O}_{2}$-Gehalt Wasser über Grund beträgt 0,4 .

- Selbst sehr hohe $\mathrm{O}_{2}$-Konzentrationen im Wasser über Grund können die durch hohen Feinmaterialanteil reduzierte Fliessgeschwindigkeit und die Folgen dieser Fliessgeschwindigkeitsreduktion nicht kompensieren [52, 53].

- Nach Rukhlov [48] gehen 75-100\% der abgelaichten O.-gorbuscha-Eier ein, wenn der Sandanteil des Laichgrundes mehr als 20\% beträgt; bei mehr als $22 \%$ Sand im Laichgrund gehen mehr als 50\% der darin eingebetteten O.-keta-Eier ein. Ähnliche Resultate erbrachten die Untersuchungen von Phillips et al. [38]. Die letzten beiden Angaben gelten für Fliessgewässer; im See können unter den dort herrschenden Zirkulationsbedingungen die Resultate bestenfalls gleich gut sein.

Werden zu den Ergebnissen, die der Versuch mit den versenkten Eiern gebracht hat, diese Aspekte mit berücksichtigt, kann man nur zum Schluss kommen, dass die Wahrscheinlichkeit für eine ungestörte Entwicklung von Seesaiblingseiern aus der Naturverlaichung im Zugersee nicht gleich Null, aber doch sehr gering ist. Die beachtliche Zahl natürlich abgelaichter (und befruchteter) Eier kann somit keinen spürbaren Beitrag an die Populationserneuerung leisten.

\subsection{Parasiten und Feinde}

Die folgende Liste basiert auf beiläufigen Erhebungen, die an ausgemessenen Fischen oder während der Erbrütung und Aufzucht von Seesaiblingen sowie am Versuchsmaterial durchgeführt wurden. Sie kann nicht als vollständig angesehen werden.

Parasiten:

Befall von Eiern: Fischschimmelähnliche Pilze, Planarien, Fischegel (Piscicola geometra) - Jugendstadien: Fischschimmelähnliche Pilze, Myxosoma cerebralis, Chondrococcus oder Cytophaga columnaris, Argulus foliaceus - Adulten: Fischschimmelähnliche Pilze. Ichthyotaenia longicollis.

Feinde von Eiern: Lota lota (Trüsche) - Adulten: Esox lucius (Hecht).

\section{Die Bewirtschaftung des Seesaiblings}

Bewirtschaften sehe ich als Tätigkeit, die bezweckt, die Produktion eines wirtschaftlichen Gutes derart zu beeinflussen, dass ein (meistens von aussen, d.h. vom Markt) gesetztes Wirtschaftsziel nachhaltig und mit möglichst geringem Aufwand verwirklicht werden kann. Für die Seesaiblingsbewirtschaftung des Zugersees heisst das. optimal wirksame Methoden auszuarbeiten, deren Anwendungen es ermöglichen. die auf 20000 Stück pro Jahr geschätzte Nachfrage des einheimischen Marktes regelmässig zu befriedigen. (Die Befriedigung nur der Nachfrage des einheimischen Marktes ergibt sich aus der hier vorweggenommenen negativen Bilanz in der Seesaiblingsbewirtschaftung und der daraus resultierenden Unterstützung durch die 
Öffentlichkeit [Kantone Zug, Schwyz und Luzern]. Diese Unterstützung ist eine Erhaltungssubvention, die durch ausserökonomische Beweggründe gerechtfertigt werden muss; es sind dies hier nur lokal zutreffende, historische Überlegungen.)

Zwei Randbedingungen sind für die Seesaiblingsbewirtschaftung am Zugersee wichtig:

- die Population kann nur künstlich aufrechterhalten werden,

- die für die künstliche Populationserneuerung verwendeten Fortpflanzungsprodukte müssen von Fischen stammen, die im Zugersee gefangen wurden: die Verwendung von fremdem oder von Teichfischen stammendem Laichmaterial würde der lokalhistorisch kolorierten, ausschliesslich während der Laichzeit betriebenen Fischerei die rechtliche Grundlage entziehen. (Die Fischerei ist während der Laichzeit verboten; sie kann zum Zweck der Entnahme von Fortpflanzungsprodukten unter bestimmten Bedingungen bewilligt werden: Verbot mit Erlaubnisvorbehalt.)

Die Verwendung von fremdem Laichmaterial bei gleichzeitiger Schonung des einheimischen Fischbestandes als Massnahme zur Förderung eines strapazierten Bestandes soll hier, obwohl unter bestimmten Voraussetzungen auch in der Seesaiblingsbewirtschaftung des Zugersees anwendbar, nicht weiter diskutiert werden. Der enge Zusammenhang zwischen diesen beiden Randbedingungen und das Bestreben, das Wirtschaftsziel mit wenig Aufwand (rationell) und nachhaltig zu verwirklichen, bedingt nicht nur den Einsatz von optimal wirksamen Besatzelementen, er setzt auch die Anwendung populationsstabilisierender fischereilicher Massnahmen voraus. Das Bestreben, die kostenintensive Saiblingsbewirtschaftung rationell zu betreiben, bedingt schliesslich technische Massnahmen, die die künstlichen und natürlichen Produktionsbedingungen verbessern.

\subsection{Besatzmassnahmen}

Besatzmassnahmen sind dann optimal wirksam, wenn das Verhältnis aus Produktionskosten für Besatzfische und deren Besatzwert minimal wird. Optimieren heisst hier also Produktionskostensenkung (einheitsbezogen) und Besatzwerterhöhung.

\subsection{Besatzwertberechnungen}

Aufgrund von Einsatzzahlen und Fangergebnissen [41] sowie aufgrund einer aus Probefängen ermittelten Altersklassenzusammensetzung der Fänge wurde der Besatzwert von Brütlingen (Brütlingseinsätze bis zum Jahr 1957) und von Vorsömmerlingen (für die Zeit von 1958 bis 1970) ermittelt. Die Zuordnung aller Fänge zu den Einsätzen ist für die früheren Jahre mit Brütlingseinsätzen bestimmt falsch; sie setzt unrichtigerweise voraus, dass damals schon kein natürlicher Beitrag an die Populationserneuerung geleistet wurde. Die Höhe des Korrelationskoeffizienten aus der Beziehung zwischen der Zeit und dem Quotienten aus Fang und Einsatz (= Besatzwert) ermöglicht jedoch, den Fehler abzuschätzen. Mit $r=0,65(\mathrm{P}=99 \%)$ ist dieser Koeffizient doppelt so hoch, wie er in einer als gesichert zu erachtenden Beziehung 
zu sein brauchte. Es scheint somit angebracht zu sein, den berechneten Besatzwert wenigstens für die letzten der in Betracht gezogenen Jahre als richtig anzusehen. Für die letzten 10 Jahre, während deren Brütlinge eingesetzt wurden, ergibt sich für Besatzfische dieses Alters ein Besatzwert von 0,0041 (pro 1000 eingesetzte Brütlinge werden 4,1 [4,1\%o] Adulte wieder gefangen); für die in den Jahren 1958-1970 eingesetzten Vorsömmerlinge beträgt der Besatzwert 0,026 (26,9\% der eingesetzten Vorsömmerlinge werden wieder gefangen).

Die Berechnung des Besatzwertes von Jährlingen basiert auf einem Markierungsversuch (für verwendete Marken siehe Abb. 13), der noch nicht als abgeschlossen gelten kann. Da die markierten Fische hinsichtlich ihrer Grösse nicht dem durchschnittlichen Fisch (auf das gleiche Alter rückberechnet) der Population entsprachen, sondern grösser waren, musste der Berechnung der noch zu erwartenden Rückmeldungen eine modifizierte Altersklassenzusammensetzung zugrunde gelegt werden, die diese unterschiedliche Wüchsigkeit (Abb. 14) und das daraus resultierende Eintreffen auf den Laichgründen in unterschiedlichem Alter berücksichtigte.

Es musste ferner in Betracht gezogen werden, dass markierungsbedingte Abgänge zu verzeichnen waren, wie sie bei der Verwendung von Kiefermarken auch von Stauffer und Hansen [55] sowie von Knecht [32] festgestellt wurden, und dass, trotz ausgesetzter Rückmeldeprämie von sFr. 2.-, die Fischer markierte Fische unbeachtet liessen.

Im Alter $6^{+}$werden wahrscheinlich die letzten markierten Seesaiblinge wieder gefangen sein. Die Wiederfanghäufigkeit kann, unter Berücksichtigung der eben genannten Einflüsse, auf 0,085 bis 0,205 geschätzt werden. Die grosse Spannweite ist auf die Tatsache zurückzuführen, dass sich die Rückmeldehäufigkeit für Fische, die im Sommer im Alter $1^{+}$im Untersee eingesetzt wurden, wo sich die Seesaiblinge während dieser Jahreszeit auch mehrheitlich aufhalten, stark von der Rückmeldehäufigkeit für im Obersee eingesetzte Fische unterscheidet. Der Unterschied zwischen den Rückmeldehäufigkeiten beider Gruppen ist gesichert. Bei konsequen-

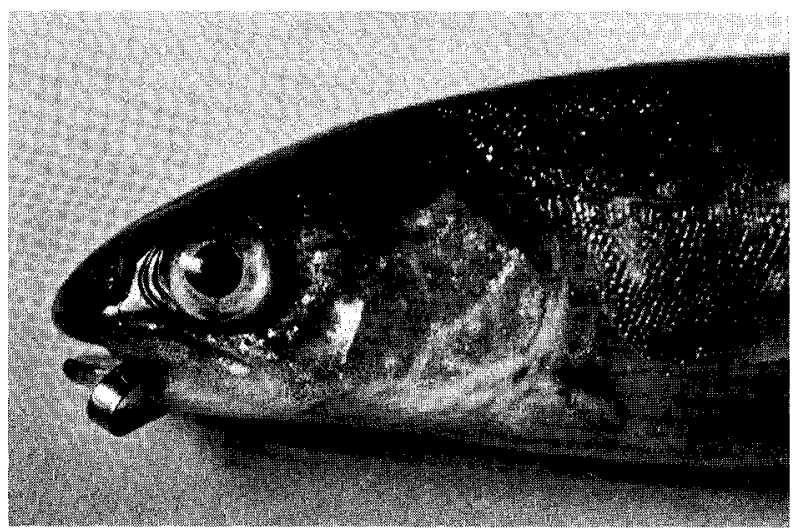

Abb. 13. Markierter $1^{+}$-Seesaibling. Marke: Individuell numeriert, Edelstahl. Hersteller: Presadom, Brive, Frankreich.

Figure 13 . Tagged arctic char, age $1^{+}$. Tags are stainless steel, individually numbered clips, made by Presadom, Brive, France. 
tem Einsatz der $1^{+}$-Fische im Untersee dürfte somit mit einem Besatzwert von 0,205 für Fische dieses Alters gerechnet werden.

\subsection{Besatzfischproduktion}

Die ersten künstlich erbrüteten Seesaiblinge wurden im Jahre 1883 in den Zugersee eingesetzt [62]. Die damals gebräuchlichen Methoden der Besatzfischproduktion wurden bis zum Jahre 1968 beibehalten und dann nur insofern abgeändert, als die Fische nicht mehr von den Fischern gestreift und die befruchteten Eier von diesen zur Brutanstalt gebracht wurden; von 1968 an ging ein Fischereiaufseher zu den einzelnen Fischern und besorgte dort die Entnahme der Fortpflanzungsprodukte und deren Transport zur Brutanstalt. Die Erbrütungserfolge waren, besonders in den letzten Jahren, schlecht; sie betrugen lediglich 60\%. Für die Pflege des Laichma-

LANGENVERGLEICH

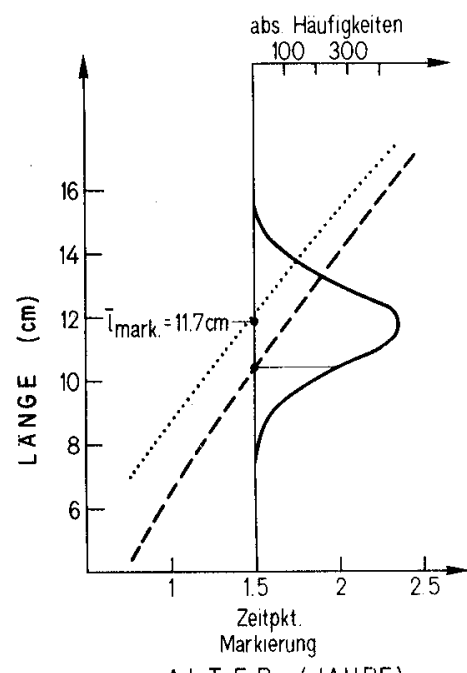

ALTER (JAHRE)
GEWICHTSVERGLEICH

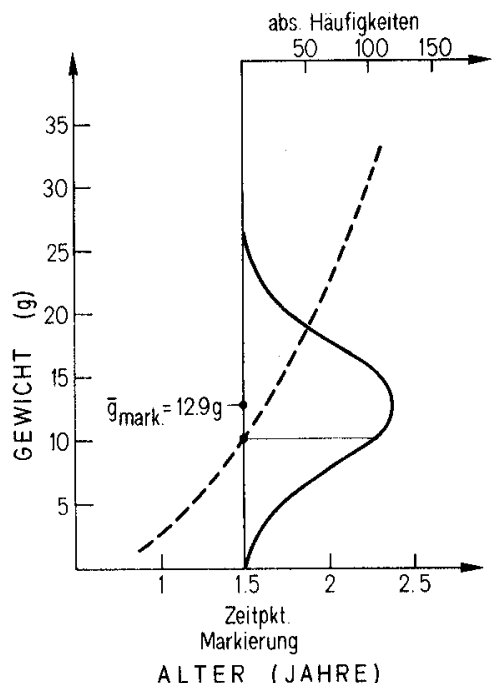

ALTER (JAHRE)
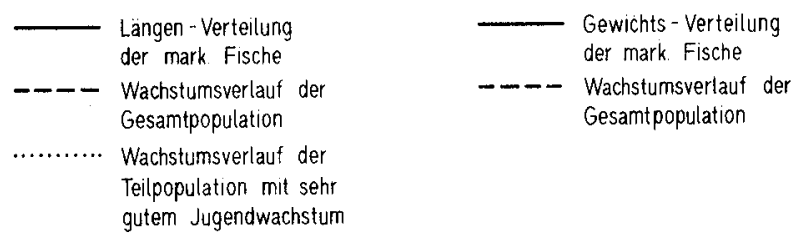

Abb. 14. Längen- und Gewichtsvergleich zwischen den im Markierungsversuch verwendeten Fischen und den «Durchschnittsfischen» im gleichen Alter.

Figure 14. Age-length and age-weight relationship of the fish from the tagging experiment as compared with the 'standard fish' of equal age. 
terials (Entfernung abgestorbener Eier ...) war ein Zeitaufwand von mindestens 4 Stunden täglich nötig.

Es war naheliegend, diese unbefriedigenden Erfolge ungeeigneten Erbrütungsbedingungen in der Brutanstalt zuzuschreiben. Als erstes wurde darum versucht, die für die Eier von Seesaiblingen aus dem Zugersee optimale Erbrütungstemperatur zu bestimmen. Verwendet wurde dazu ein umgebauter Brutschrank, bei dem jedes der 10 Brutsiebe mit unterschiedlich warmem Wasser zwischen 3 und $12^{\circ} \mathrm{C}$ beschickt werden konnte. Zur Beurteilung des Erfolges dienten einerseits die Abgänge. anderseits die am Ende der Dottersackresorption bestimmten Gewichte und Längen der Brütlinge. Die Ergebnisse sind aus den Abb. 15 und 16 ersichtlich.

Erstaunlich ist, dass die Fische, die aufgrund der Abgänge als unter optimalen Bedingungen erbrütet angesehen werden müssen, die geringsten oder nahezu geringsten Gewichte hatten. Die Erscheinung kann nur dadurch erklärt werden, dass unter guten Bedingungen erbrütete Fische aktiver sind als andere und einen entsprechend grösseren Teil ihrer Reserven im Betriebsstoffwechsel umsetzen. Der Zeitpunkt der Messung am Ende der Dottersackresorption (das ohnehin schwer feststellbar ist) muss demzufolge als ungeeignet angesehen werden. Wägungen im Zeitpunkt des Schlüpfens hätten allenfalls zutreffendere Ergebnisse gebracht. Die durch schwierig interpretierbare Resultate verursachte Ungewissheit hinsichtlich der Qualität des verschieden warmen Wassers für die Erbrütung zwang zur Fortsetzung des Versuches während einer drei Monate dauernden Aufzuchtsperiode. Die Ergebnisse dieses Kontrollversuches (Abb. 17, 18) bestätigen indessen, dass für Eier von Seesaiblingen aus dem Zugersee bei Wassertemperaturen um $6{ }^{\circ} \mathrm{C}$ optimale Erbrütungsbedingungen herrschen.

Die Ergebnisse beider Versuche führen zum Schluss, dass nicht die Erbrütungsbedingungen in der Anstalt, wo das Wasser während der Erbrütungsperiode Temperaturen um 5 bis $6^{\circ} \mathrm{C}$ aufweist, die grossen Abgänge verursacht haben können.

Die Erbrütung des Laichmaterials getrennt nach Fischern, die es geliefert hatten, zeigte dann, dass die Behandlung der Elterntiere zwischen dem Fang und dem

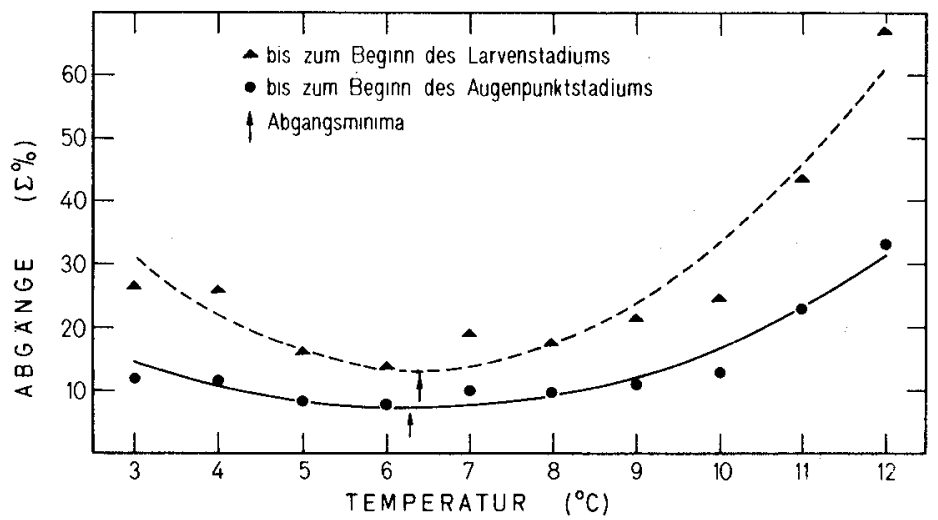

Abb. 15. Abgänge während der Erbrütung in Abhängigkeit von der Frbrütungstemperatur.

Figure 15. Hatchery losses in relation to incubation temperature: $\Delta$ up to larval stage, $\boldsymbol{\omega p}$ to eyed stage.

minimum loss. 


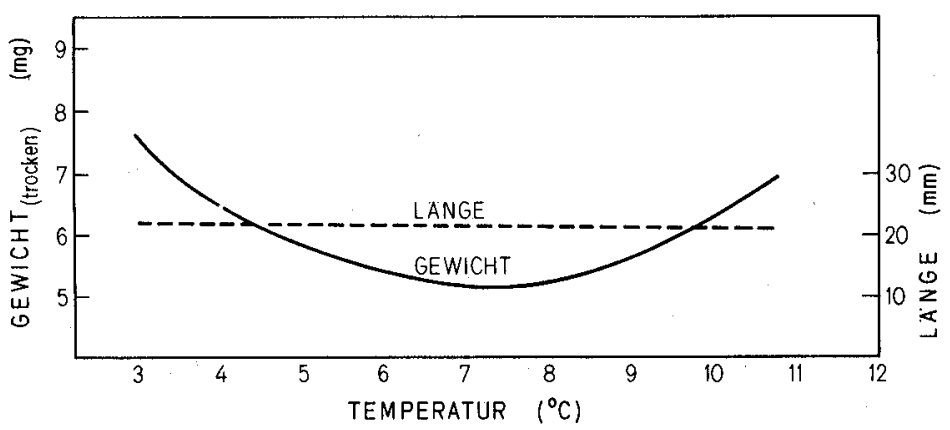

Abb. 16. Länge und Gewicht der bei verschiedenen Temperaturen erbrüteten Seesaiblinge am Ende der Dottersackresorption.

Figure 16. Length (----) and weight $(-$ ) of arctic chars at the end of yolk sac resorption, as compared to incubation temperature.

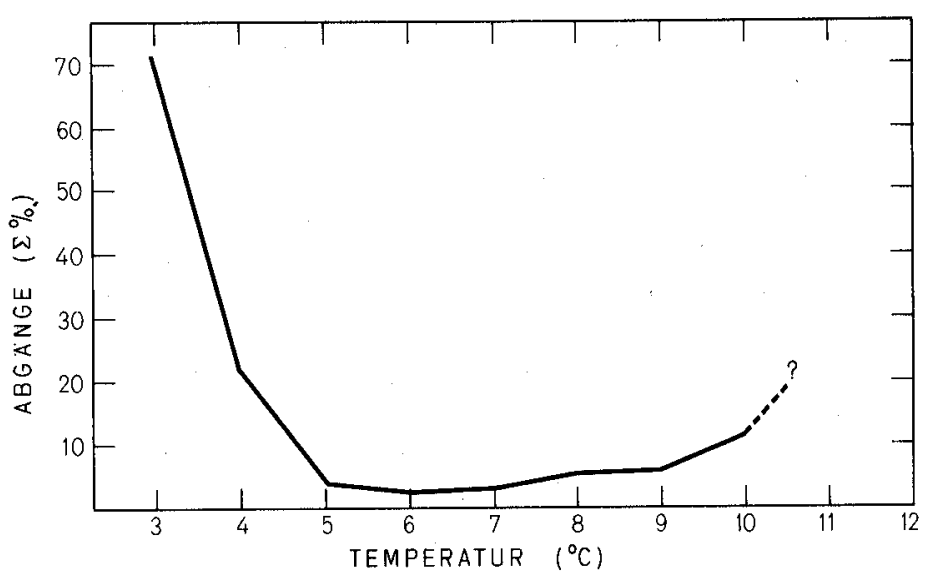

Abb. 17. Abgänge während einer dreimonatigen Aufzuchtperiode unter seeähnlichen Bedingungen in Abhängigkeit der Erbrütungstemperatur.

Figure 17. Losses during a three-month rearing period under lake-like conditions, as compared to egg incubation temperature.

Streifen bei verschiedenen Fischern unzulänglich war (tägliche Entnahme aus den Behältern zur Überprüfung des Reifegrades; Kontrolle, ob die für den Verkauf vorgesehenen Fische schon gestreift wurden; Rückversetzen der Fische in die Behälter ...). Durch das heute praktizierte Streifen gleich nach dem Fang in der Brutanstalt, wo die meisten Fischer nach dem Heben der Netze vorbeikommen, konnten die Abgänge wiederholt auf weniger als 10\% reduziert werden.

Im Jahre 1941 empfahl Steinmann [56] den Einsatz von Seesaiblingsvorsömmerlingen; seit 1958 werden seine Empfehlungen befolgt. Die Produktion von Vorsömmerlingen geschieht in Eternit-Rundtrögen. Die bisher erreichten Aufzuchtsresultate sind allerdings sehr schlecht: im Jahre 1973 wurde ein Erfolg von nur 13\% erzielt [33]. Die Seesaiblinge zeigen im Rundtrog das gleiche Verhalten, wie es für Forellen 


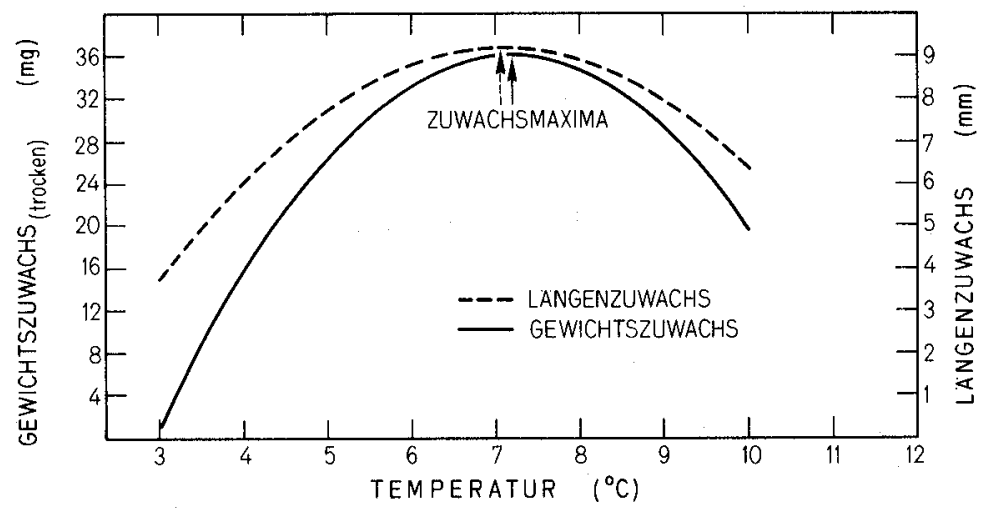

Abb. 18. Zuwachsleistungen während der dreimonatigen Aufzuchtperiode. Figure 18. Length and weight increases during a three-month rearing period.

von Roth und Geiger [45] beschrieben wurde. Die Fische halten sich am Trogboden in Futterresten und Exkrementen auf; sie werden dort von Parasiten befallen, bald apathisch und neigen zu Fressunlust. Die naheliegendste Therapie, den abgemagerten Fischen vermehrt Futter zuzuführen, verschlimmert die Situation, führt im Extremfall zu Kiemenverstopfungen und häufig zur bakteriellen Kiemenkrankheit (Cytophaga columnaris). Roth und Geiger [45] haben die Anwendung von Siebeinsätzen vorgeschlagen, die, im Rundtrog schwimmend, die im Sieb gehaltenen Fische ans Schwimmen und Fressen im Freiwasser gewöhnen. Ein erster Versuch damit zeigte anfänglich auch in der Brutanstalt Walchwil die gewünschten Resultate. Er musste allerdings vorzeitig abgebrochen werden: der Algenbehang an der Unterseite des Siebtellers hatte die Zirkulation und damit das Wegspülen von Exkrementen und Futterresten verhindert. Die Anwendung des Siebtellers in filtriertem Wasser (Installation eines Filters im Jahre 1975) dürfte bessere Resultate bringen.

Wie für die Erbrütung wurde auch für die Vorsömmerlingsaufzucht versucht, die ideale Wassertemperatur zu bestimmen. Der Versuch wurde in 40-1-Behältern durchgeführt, in denen das Wasser durch Thermostaten auf den gewünschten Temperaturen $\left(8,10,12,14^{\circ} \mathrm{C}\right.$; Kontrolle $=$ Betriebstemperatur der Anstalt: $\left.5-7^{\circ} \mathrm{C}\right)$ gehalten wurden. Wie aus der Tabelle 8 hervorgeht, zeigten die Fische im $10^{\circ} \mathrm{C}$ Becken die grössten Zuwachsleistungen. Das Abgangsminimum (aufsummiert) wurde im $12^{\circ} \mathrm{C}$-Becken festgestellt.

Temperaturen um $10^{\circ} \mathrm{C}$ entsprechen der Betriebstemperatur der Brutanstalt Weesen während der Seesaiblingsaufzucht [5] und derjenigen der Anstalt St. Moritz während der Forellenaufzucht [40]. Schwedische Aufzuchtversuche haben für die Seesaiblingsaufzucht ähnliche optimale Temperaturen erbracht [19]. Grundsätzlich ist es möglich, Wasser dieser Temperatur in der Anstalt Walchwil zu mischen: die Anstalt verfügt über zwei Ansaugstutzen ( $1 \mathrm{~m}$ und $30 \mathrm{~m}$ tief), von denen warmes und kaltes Wasser bezogen werden könnte. Das Pumpsystem müsste fürs Mischen allerdings abgeändert werden.

Neben der Anwendung von Siebtellern und der Anpassung der Wassertemperatur 
an die Bedürfnisse der Fische könnte die Fütterung von Kunstfutter eine weitere Verbesserung der Aufzuchterfolge bringen (in der grössten öffentlichen Anstalt von Britisch-Kolumbien, Kanada, in Wardner (Kootenay Trout Hatchery) werden alle dort produzierten Salmoniden-Besatzfische ausschliesslich mit Kunstfutter an- und weitergefüttert; Abgänge bis zum Einsatz als Fingerlinge: ca. 20\%). In Anstalten, in denen gute Produktionsvoraussetzungen herrschen (z. B. Faulensee BE), werden regelmässig Aufzuchterfolge von $80 \%$ erreicht.

Seit Herbst 1972 besitzt die Fischereiverwaltung des Kantons Zug ein Netzgehege; die beiden Netztaschen haben ein Volumen von je $48 \mathrm{~m}^{3}$, die Maschenweiten betragen 4,25 bzw. $6 \mathrm{~mm}$. Seit drei Jahren werden darin zweisömmerige Seesaiblinge aufgezogen. Der beste bislang erzielte Aufzuchterfolg (vom Vorsömmerlingsalter an) betrug $85 \%$. In jedes Netzabteil können bis zu 20000 Seesaiblingsvorsömmerlinge eingesetzt werden: die Höhe der Abgänge wird von der Besatzdichte wenig beeinflusst; bei grösserer Dichte sind die Zuwachsleistungen jedoch bedeutend geringer (101 mm Totallänge und $9,9 \mathrm{~g}$ Gewicht bei 81 Wasser je Fisch bzw. 57,2 mm und 1,42 g bei 1,51 Wasser je Fisch; Aufnahmedaten 20. bzw. 24. April).

\subsection{Produktionskosten und Nutzen-Aufwand-Vergleiche}

In der Tabelle 9 sind die Ergebnisse der für die verschieden alt eingesetzten Besatzfische durchgeführten Produktionskostenanalysen zusammengefasst. Diejenigen Angaben, die nicht selbst ermittelt werden konnten, stammen von Frei [22] und von Roth und Geiger [45]. Aus der Tabelle 10 sind die Nutzen-Aufwand-Relationen für die unter verschiedenen Bedingungen produzierten Brütlinge, Vorsömmerlinge und Jährlinge ersichtlich. Beide Tabellen zeigen deutlich, wie sehr die Erbrütungs- bzw. Aufzuchterfolge den Umfang der finanziellen Aufwendungen für Besatzmassnahmen zu beeinflussen vermögen.

\subsection{Fischereiliche Massnahmen}

Die Seesaiblingsfischerei im Zugersee muss so betrieben werden, dass

- Fische, die sich nicht effektiv fortpflanzen konnten, vom Fang ausserhalb der Laichzeit ausgeschlossen bleiben und

- eine aus mindestens zwei fortpflanzungsfähigen Jahrgängen bestehende befischte Population aufgebaut wird.

Zur ersten Forderung: Sie führt, in letzter Konsequenz, zum Verzicht jeglicher Seesaiblingsfischerei ausserhalb der Laichzeit: selbst wenn Seesaiblinge im Zugersee unter natürlichen Bedingungen zum Laichen kommen, haben sie, nach den im Kapitel über die Fortpflanzung gezogenen Schlüssen, nicht zur Populationserneuerung beigetragen; dies geschieht erst dann, wenn ihre Fortpflanzungsprodukte unter günstigen, künstlich beeinflussten Bedingungen verwertet werden. Die Seesaiblingsfischerei im Zugersee, wie sie heute betrieben wird, erbringt keine Fische, deren Fortpflanzungsprodukte altersbedingt unbrauchbar wären.

Zur zweiten Forderung: Die Altersklassenzusammensetzung des Laichfischfanges 1971 zeigt, dass, wenigstens zeitweilig, die Population vorwiegend aus Fischen 
besteht, die zum grossen Teil erstmals laichreif sind $\left(2^{+}: 5,88 \%, 3^{+}: 77,64 \%, 4^{+}\right.$: $7,06 \%, 5^{+}: 5,88 \%, 6^{+}: 2,35 \%, 7^{+}: 1,18 \%$ ). In Seen, in denen die Produktionsbedingungen für Seesaiblinge nahezu pessimal sind, ist es denkbar, dass die konsequente Nachfolge jüngerer Jahrgänge unterbrochen wird; Gründe hierzu gibt es viele: schlechte Bedingungen beim Laichfischfang, Versagen der Aufzuchteinrichtungen, ungünstige Bedingungen beim Einsatz, schlecht gewählte Einsatzorte ... Die nachhaltige Verwirklichung des Wirtschaftszieles setzt nun eine stabile Population voraus, die um so eher zu realisieren ist, je grösser die Anzahl fortpflanzungsfähiger Jahrgänge in ihr ist (der Zusammenbruch einer Population hängt vom Alter der fortpflanzungsfähigen Tiere, von der Anzahl fortpflanzungsfähiger Generationen und von der Anzahl sich folgender, nachwuchsfreier [oder -armer] Jahrgänge ab). Die Forderung wird dann erfüllt, wenn ein genügend grosser Teil der erstmals laichreifen und grundsätzlich fangfähigen Fische vom Fang nicht erfasst wird.

Die Bestimmung der Maschenweite, deren Anwendung die Erfüllung der zweiten Forderung verwirklichen kann, basiert auf Probefängen mit Netzen verschiedener Maschenweite und auf der Berechnung von Alter-Längen-Beziehungen. Die Ergebnisse dieser Erhebungen führen zu den in Tabelle 11 zusammengestellten berechneten relativen Häufigkeiten der verschiedenen Altersklassen in den Fängen mit Netzen verschiedener Maschenweite und zu der in Abb. 19 ausgerundet dargestellten Fanganteile-Alter-Maschenweite-Beziehung.

Da ein der zweiten Forderung entsprechendes ausgewogenes $2^{+}: 3^{+}$-Verhältnis nicht zu realisieren ist, muss die aus zwei fortpflanzungsfähigen Generationen bestehende befischte Population aus $3^{+}$- und $4^{+}$-Fischen aufgebaut werden. Die partielle Schonung der $3^{+}$-Fische kann durch die Anwendung von Netzen mit $35-36 \mathrm{~mm}$ Maschenweite erreicht werden. Die teilweise Schonung der erstmals laichreifen Fische und deren Fang in höherem Alter führt zwangsläufig auch zu einem erhöhten Laichertrag; dem (meines Erachtens in dieser kritischen Situation irrelevanten) Konsumentenwunsch nach kleinen Fischen kann leider nicht entsprochen werden.

\subsection{Technische Massnahmen}

Technische Massnahmen wurden als Vorkehrungen zur Verbesserung der natürlichen und künstlichen Produktionsbedingungen mit dem Ziel, die Seesaiblingsbewirtschaftung rationeller (ökonomischer) zu gestalten, definiert. $\mathrm{Zu}$ ihnen gehören alle Verbesserungen an den Produktionseinrichtungen, die im Laufe dieser Untersuchungen empfohlen wurden und zum Teil schon realisiert sind (Netzgehege für die Jährlingsproduktion, Siebeinsätze für die Vorsömmerlingsproduktion, Änderungen am Pumpsystem für die Wasseraufbereitung, Filteranlage zur Verhinderung der Algenbeläge an Trögen und Siebeinsätzen ...). Zu ihnen gehören aber auch Gewässerschutzmassnahmen, dank deren der See schliesslich in denjenigen Zustand gebracht werden soll, der eine naturnähere Seesaiblingsbewirtschaftung ermöglicht (die Prognosen, dieses Ziel innert nützlicher Frist zu erreichen, sind allerdings wegen der besonderen hydrologischen Verhältnisse und wegen des heutigen Standes in der Errichtung von Schutzanlagen nicht sehr günstig).

Eine technische Massnahme besonderer Art stellt die Bekiesung der Laichgründe dar. Sie wurde sicher schon 1844 praktiziert (1844: Gründung der Fischergesell- 


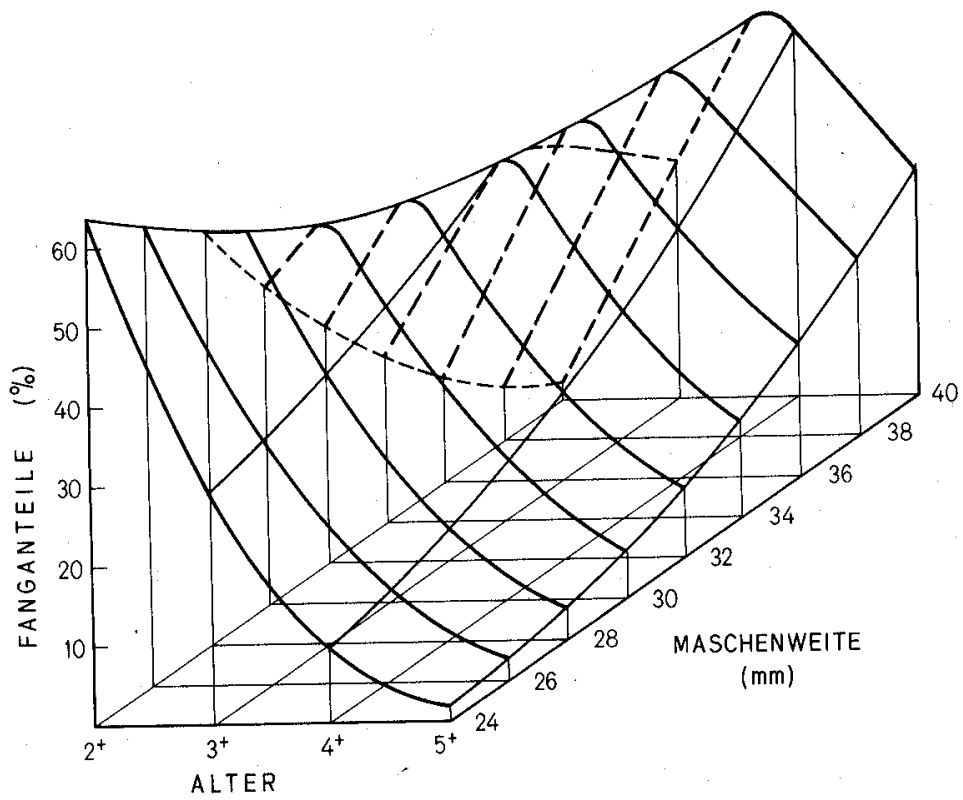

Abb. 19. Fanganteile-Alter-Maschenweite-Beziehungen (ausgerundete berechnete Werte).

Figure 19. Relationship between mesh size, age of the fish and catch percentage (rounded calculated values).

schaft Walchwil; eines ihrer Ziele bestand in der kooperativ besser zu bewältigenden Bekiesung [31]) und zeugt von einem für die damalige Zeit erstaunlichen Verständnis für fischereibiologische Belange. Da damals die Voraussetzungen für eine natürliche Eientwicklung noch bestanden [66], muss sie als eine Massnahme zur Verbesserung der Fischerei (Konzentration der Fische auf kleinen, leicht befischbaren Flächen) und als Mittel angesehen werden, mit dem sich die aus der Gesellschaft ausgeschlossenen Fischer (Anlage eigener, neuer Laichgründe) die Möglichkeit zum Seesaiblingsfang schufen [31].

Seit der Verbauung der Wildbäche (Fliessgeschwindigkeitsreduktion, Stabilisierung der Hänge und der Sohle, Reduktion des Eintrags an grobem Geschiebe ...) und seit der eutrophiebedingten Intensivierung der autochthonen Sedimentation stellt sie, wie aus der Abb. 12 hervorgeht, eine elementare Voraussetzung für den Laichfischfang dar.

Dank photographischen Erhebungen war es möglich, die oft geäusserte Befürchtung, dass Bekiesungen an sehr steilen Hängen nicht zu stabilen Laichgründen führen könne, zu entkräften (siehe auch Doerfel [14] über Laichgründe auf Hängen von ca. $30^{\circ}$ Neigung). Der steilste Hang, auf dem ein stabiler Laichgrund photographiert wurde, war um $63^{\circ} 30^{\prime}$ geneigt.

Wegen der unterschiedlichen Zirkulations- und Sedimentationsverhältnisse war die Bestimmung einer allgemeingültigen Bekiesungsfrequenz nicht möglich. Unnütze Bekiesungen noch gut erhaltener Laichgründe können darum wohl nur durch periodische photographische Zustandserhebungen, wie im Rahmen dieser Arbeit 
für alle Laichgründe durchgeführt, vermieden werden. Bislang wurden die heute noch bestehenden Laichgründe unabhängig von ihrer Qualität ungefähr alle 3 Jahre bekiest. Es werden dabei etwa 20 bis $30 \mathrm{~m}^{3}$ Kies von 3 bis $8 \mathrm{~cm}$ Korngrösse aus einem verankerten Kahn über die durch Fixpunkte auf dem Land genau eingemessenen Laichgründe schaufelweise versenkt. Bekiesungen mit einem Klappschiff haben die gewünschten Ergebnisse nicht erbracht. Die nach herkömmlicher Art ausgeführte Bekiesung eines Laichgrundes kostet etwa Fr. 750.-.

Seit einiger Zeit meiden die Seesaiblinge zum Teil auch gut unterhaltene Laichgründe. Die Erscheinung ist darauf zurückzuführen, dass bei ungenügend wirksamer Herbstzirkulation das Wasser über den Laichgründen ungenügende Sauerstoffkonzentrationen aufweist. Die Verlegung der Laichgründe in Zonen mit besseren $\mathrm{O}_{2}$ Bedingungen (über das in der Laichzeit manchmal rudimentär noch ausgebildete metalimnische $\mathrm{O}_{2}$-Minimum) könnte eine Abhilfe bieten [47]. Die Anlage neuer Laichgründe auf noch unbefestigten Böden wäre allerdings sehr teuer und würde mit Bestimmtheit zur Frage nach dem Sinn der Fortsetzung der Seesaiblingsfischerei im Zugersee führen.

\subsection{Die Bilanz der Seesaiblingsbewirtschaftung}

Der Gesamtaufwand in der Seesaiblingsbewirtschaftung setzt sich aus den Kosten für Besatzmassnahmen, fischereiliche Massnahmen und technische Massnahmen zusammen. Seine Höhe wird vorab von den Kosten für Besatzfische und für die Bekiesung bestimmt; er hängt somit wesentlich von der Differenz zwischen aktuellen und optimalen Produktionsbedingungen im See ab. Die Nutzungskosten variieren dagegen in Abhängigkeit der Produktionsbedingungen wenig. Die Aufwendungen schwanken zwischen Fr. 40000.- und Fr. 290000.-, je nachdem welches Ziel (formuliertes Wirtschaftsziel [20000 Stück] bzw. Durchschnittsertrag der letzten 10 Jahre [8400 Stück] bzw. bestmögliche Ausnützung der Produktionskapazität) angestrebt wird und welche Produktionsverfahren angewandt werden.

Der Gesamtertrag beträgt etwa Fr. 50000.-, wenn das nachfragegerechte Ziel verwirklicht wird. Er erreicht lediglich Fr. 21000 -, $^{-}$wenn die gleichen Erträge wie in den letzten 10 Jahren erzielt werden.

Die Bilanz ist auf jeden Fall negativ. Wenn versucht wird, das nachfragegerechte Ziel mit optimal produzierten Besatzfischen zu verwirklichen, so ist mit einem durch Erträge gedeckten Aufwandanteil von 72,3\% (100\%=Fr. 69000.-) zu rechnen. Werden nach herkömmlichen Verfahren produzierte Fische in derjenigen Menge als Besatzelemente verwendet, dass der durchschnittliche Ertrag der letzten 10 Jahre weiterhin realisiert wird, so beträgt dieser Anteil lediglich $16 \%(100 \%=$ Fr. $131000 .-)$. Das Defizit wird zum überwiegenden Teil von der Öffentlichkeit getragen, deren Leistungen als Erhaltungssubventionen, basierend auf ausserökonomischen, nur lokal gültigen, historischen Überlegungen, zu betrachten sind [18].

\section{Zusammenfassung}

Die Erträge der lokalhistorisch bedeutenden Seesaiblingsfischerei des Zugersees sanken in den letzten 70 Jahren von etwa 100000 Stück auf etwa 5000 Stück pro 
Jahr. Im Bestreben, die kontinuierliche Ertragsabnahme zu bremsen oder gar in eine Ertragszunahme umzuwandeln, wird die Biologie der Seesaiblinge im Zugersee untersucht. Auf den gewonnenen Erkenntnissen aufbauend, wurden neue Bewirtschaftungsmethoden erarbeitet.

Es wird festgestellt, dass die Seesaiblinge (Salvelinus alpinus L.) des Zugersees das eutrophiebedingt grössere Nahrungsangebot auszunützen vermögen, dass dagegen aber die Wahrscheinlichkeit für eine natürliche Fortpflanzung unter den heute im See herrschenden Bedingungen sehr gering ist.

Wichtigste Massnahmen zur Sanierung der Ertragsverhältnisse sind eine qualitativ und quantitativ verbesserte Besatzwirtschaft (Einsatz von Jährlingen), der Einsatz grobmaschigerer (35/36 mm) Netze zum Zweck der Populationsstabilisierung, der Verzicht auf die Fischerei ausserhalb der Laichzeit und, eventuell, die Neuanlage von Laichgründen in weniger tiefen Zonen mit besseren $\mathrm{O}_{2}$-Bedingungen.

Die vorgeschlagenen Massnahmen sind kostenintensiv. Das von der Öffentlichkeit getragene Defizit kann durch ausserökonomische, lokal gültige, historische Überlegungen gerechtfertigt werden.

\section{SUMMARY}

Biology and management of the arctic char (Salvelinus alpinus L.) in Lake Zug (Switzerland)

During the past 70 years the yields of the arctic char fishery in Lake Zug dropped from some 100,000 to 5,000 fish per year. The aim of this work was to develop methods for retarding and perhaps reversing this downward trend.

To serve as a basis for new methods of fish stock management, the biology of the arctic char was investigated. It was found that the fish in the eutrophic lake consume the increased food supply.

It could also be shown that the arctic char in Lake Zug are now unable to reproduce naturally.

The following measures for improving the yield are recommended:

- intensified stocking (yearlings if possible),

- the use of gillnets with larger mesh sizes $(35 \mathrm{~mm})$

- and eventually the dislocation of the artificially created and maintained spawning grounds (sole function today: catching areas) to more shallow zones with better oxygen conditions.

The measures for improving the fish stock are costly. Even with maximum yields the cost-benefit balance of the arctic char management is negative. Only the historical significance of the arctic char fishery in Lake Zug can justify the considerable expenditure of public funds.

\section{RESUMÉ}

Biologie et aménagement de l'omble chevalier (Salvelinus alpinus L.) dans le lac de Zoug (Suisse)

Pendant ces 70 dernières années, le rendement de la pêche de l'omble chevalier (Salvelinus alpinus L.) du lac de Zoug a diminué d'environ 95\%, passant ainsi de 100000 à 5000 poissons par an. Le but de ce travail consiste à trouver le moyen de freiner cette diminution ou, même éventuellement, de réaugmenter les prises annuelles.

La biologie de l'espèce est étudiée, elle servira de base pour de nouvelles mesures d'aménagement. On constate, d'une part, que le poisson est capable d'exploiter la production accrue de nourriture due à l'eutrophisation du lac. On doit, d'autre part, conclure qu'il est très improbable que l'espèce puisse encore se reproduire naturellement.

Pour augmenter le rendement annuel, on recommende l'application des mesures d'aménagement suivantes:

- intensifier le renouvellement artificiel du stock par l'immersion de poissons d'un an;

- stabiliser la population en créant au moins deux générations reproductrices, par l'utilisation de filets dont les mailles ont 35 à $36 \mathrm{~mm}$; 


\section{Ch. Ruhlé}

renoncer à la pêche en dehors de la période de reproduction;

- déplacer, éventuellement, certaines frayères dans des zones moins profondes où les conditions respiratoires sont plus favorables.

Les mesures recommendées sont coûteuses. Le déficit de la pêche de l'omble chevalier, couvert par les fonds publics, ne peut être justifié que par le rôle extraordinaire, voire historique, de ce poisson du lac de Zoug.

\section{VERDANKUNGEN}

Diese Arbeit basiert weitgehend auf einer in den Jahren 1971 bis 1976 am Institut für Gewässerschutz und Wassertechnologie der ETH und der EAWAG durchgeführten Dissertation. Mein Dank gilt vorab den Herren Dr. W. Geiger und Prof. Dr. W. Stumm von der EAWAG, Herrn Prof. Dr. H. Leibundgut, Institut für Waldbau ETH, den Herren E. Feuz und Dr. H. Schwarzenbach von der Schweizerischen Stiftung für Alpine Forschungen und Herrn R. Keiser, Fischereiaufseher in Walchwil. Sie alle haben entscheidend zum Gelingen der Arbeit beigetragen.

\section{LITERATURVERZEICHNIS}

1 Aldridge, P.F., Wicket, W.P., und Brett, J.R.: Some effects of temporary exposure to low dissolved oxygen levels on Pacific salmon eggs. J. Fish. Res. Bd Canada 15, 229-250 (1958).

2 Alm, G.: Connection between maturity, size and age in fish. Rep. Inst. Freshw. Res. Drottningholm 40. 4-145 (1959).

3 Allen, K.R.: A methode of fitting growth kurves of the von Bertalanffy type to observed data. J. Fish. Res. Bd Canada 23 (2), 163-179 (1966).

4 Alvord, W.: Validity of age determination from scales of brown trout, rainbow trout and brook trout. Trans. Am. Fish. Soc. 83 (1), 91-103 (1954).

5 Baelli, W., mündliche Mitteilung (1975)

6 Bagenal, T. B.: The ecological and geographical aspects of the fecundity of the plaice. J. mar. biol. ass. U.K. 46, 161-186 (1966).

7 Bagenal, T.B.: A short review of fish fecundity. In: Gerking, S.D. (Ed.): The biological basis of IBP-Program, Blackwell Sci. Publ. Oxford and Edinburgh 1967.

8 Behnke, R.: The salmonid fishes of recently glaciated lakes. J. Fish. Res. Bd Canada 29 (6), 639-671 (1972).

9 Bertalanffy, von, L.: A quantitative theory of organic growth. Hum. Biol. 10, 181-213 (1938).

10 Bigelow, H. B.: Genus Salvelinus Richardson (1836). Sears found.

11 Brafield, A.E.: Quelques facteurs affectant la teneur en oxygène des eaux interstitielles littorales. Vie et Milieu 16, 889-897 (1965).

12 Brutschy, A.: Monographische Studien am Zugersee. Arch. Hydrobiol. 8, 43-107 (1913).

13 Dahl, K.: Studies of trout and trout-waters in Norway. Salm. Trout Mag. 17, 58-79 (1918), ibid. 18, 16-33 (1919).

14 Doerfel, H.J.: Untersuchungen zur Problematik der Saiblingspopulationen

J. Eine Schlitten-Dredge. Sep. Int. Rev. Ges. Hydrobiol. Hydrogr. 29, 3, 4 (1933).

16 Eltringham, S.K.: Life in mud and sand. The English Universities Press Ltd., London 1971.

17 Engelhardt, W.: Was lebt im Tümpel, Bach und Weiher? Kosmos Naturführer. Kosmos Verlag, Franckhsche Verlagsbuchhandlung, Stuttgart 1967.

18 Expertengruppe, vom Bundesrat eingesetzte: Allgemeine Überprüfung der Bundessubventionen. Bundeskanzlei. Bern 1966.

19 Faber-Ewos, Futtermittel, mündliche Mitteilung.

20 Fabricius, E.: Aquarium observations of the spawning behaviour of the char, Salmo alpinus L. Rep. Inst. Freshw. Res. Drottningholm 34, 14-48 (1953).

21 Fabricius, E., und Gustafson, K.J.: Further aquarium observations on the spawning behaviour of the char, Salmo alpinus L. Rep. Inst. Freshw. Res. Drottningholm 35. 58-104 (1954). 
22 Frei, A.: Approximativer Kostenaufwand für Rötel- und Hechtaufzucht, unveröffentlicht (1973).

23 Friend, G.F.: Subspeciation in British chars. Syst. Ass. Publ. 3, 121-129 (1959)

24 Glass, G.E., und Poldoski, J.E.: Interstitial water components and exchange across the water sediment interface of Western Lake Superior. Verh: int. Ver. Limnol. 19, 405-420 (1975).

25 Grainger, E.H.: On the age, growth, migration, reproductive potential and feeding habits of the arctic char (Salvelinus alpinus) of Frobisher Bay, Baffin Island. J. Fish. Res. Bd Canada 10 (6), 326370 (1953).

26 Grimaldi, E.: Lago Maggiore effects of exploitation and introductions on the salmonid community J. Fish. Res. Bd Canada 26 (6), 777-785 (1972).

27 Haempel, O.: Fischereibiologie der Alpenseen. In: Thienemann, A. (Hrsg.): Die Binnengewässer, Bd. 10. Stuttgart 1930.

28 Hayes, F.R., und Macaulay, M.A.: Lake water and sediment, V: Oxygen consumed in water over sediment cores. Limnol. Oceanogr. 4 (3), 291-297 (1959).

29 Hoar, W.S.: Smolt transformation: evolution, behaviour and physiology. J. Fish. Res. Bd Canada 33 (5), 1234-1252 (1976).

30 Iten, A.: Die ehemaligen Fischereirechte der Stadt Zug im Zugersee. Diss. Freiburg. Kalt-Zehnder, Zug 1920

31 Iten, A.: Die Rötelfischerei im Zugersee. Kunz, Pfäffikon 1933.

32 Knecht, A.: Der Einfluss von Markierungen auf das Wachstum von Fischen bei unterschiedlichem Futterangebot, unveröffentlicht (1974).

kommission über die Konkordatsfischerei, polycopiert (1889-1975).

34 Muus, B.J., und Dahlstroem, P.: Süsswasserfische. BLV, München 1968 Nikolsky, G.V.: Spezielle Fischkunde. VEB Deutscher Verlag der Wissenschaften, Berlin 1957. Phillips, R.W., Lantz, R. L., Claire, E.W., und Moring, J.R.. Som . Soc. 104 (3), 461-466 (1975). emergence of Coho salmon and Steelhead trout fry. Trans. Am. Fish. Soc. Limnol. 19, 1313-1318 (1975).

40 Ratti, P., mündliche Mitteilung. Fischerei-Aufseher-Kurs, 1975.

41 Regierungsrat des Kantons Zug: Rechenschaftsberichte. Staatskanzlei, Zug (bis 1974)

42 Reisinger, E.: Zum Saiblingsproblem. Carinthia II 63 (2), 74-102 (1953).

43 Rensch, B.: Das Prinzip geographischer Rassenkreise und das Problem der Artbildung. Berlin 1929 Roth, $\mathrm{H}_{\text {, }}$ und Geiger, W.: Die Fortpflanzung der Forelle im Bach und in der Brutanstalt. Schweiz. Fisch. Ztg., Sep. $2+3$ (1961). Roth, H., und Cei
Insp. 25 (1968). Diss. ETH 5809, 221 S. Aku-Fotodruck, Zürich 1976.

47 Ruhlé, Ch.: Maintien de la population des ombles chevaliers (Salvelinus alpinus L.) dans le lac de Zoug par l'entretien artificiel des frayères et par des repeuplements de poissons d'âges divers. Actes du Deuxième Congrès Européen des Ichthyologistes, Paris, 8-14 septembre 1976.

48 Rukhlov, F.N.: Materials characterizing texture and the materials in the spawing grounds and redds of the Pink salmon [Oncorhynchus gorbuscha (Walb.)] and the Autumn chum [Oncorhynchus keta (Walb.)] of Sakhalin. Probl. Ichth. 9 (5), 635-644 (1969).

49 Savvaitova, K.A.: Homologous variation in char species of the genera Salvelinus (Nilsson) Richardson and Cristivomer Gill and Jordan. Probl. Ichth. 1, 18-33 (1969).

50 Schindler, O.: Unsere Süsswasserfische. Kosmos, Franckh'sche Verlagsbuchhandlung, Stuttgart 1968.

51 Segestrale, C.: Über skalimetrische Methoden zur Bestimmung des linearen Wachstums bei Fischen. Acta Zool. fennica I (1933).

52 Shumway, D.L., Warren, C.E., und Dudoroff, C.: Influence of oxygen concentration and water movement on the growth of Steelhead trout and Coho salmon embryos. Trans. Am. Fish. Soc. 93 (4), 342-356 (1964) 
53 Silver, S.J., Warren, C.E., und Dudoroff, C.: Dissolved oxygen requirements of developping Steelhead trout and Chinook salmon embryos at different water velocities. Trans. Am. Fish. Soc. 92, 327-343 (1963).

54 Southward, A.J.: Improved methods of sampling post-larval young fish and makroplankton. J. mar. biol. Ass. U.K. 50, 689-712 (1970).

55 Stauffer, T.M., und Hansen, M.J.: Mark retention, survival and growth of jaw-tagged and fin clipped rainbow trout. Trans. Am. Fish. Soc. 98, 225-229 (1969).

56 Steinmann, P.: Ủber Verbesserungen in der Rötelzucht. Siehe Konkordatsbericht (1941).

57 Steinmann, P.: Experimentelle Untersuchungen über die Wüchsigkeit des Seesaiblings. Schweiz. Fisch. Ztg. 9, 1-9 (1942).

58 Surbeck, G.: Beitrag zur Kenntnis der Fischereiverhältnisse im Zugersee. Schweiz. Fisch. Ztg. 3 (1915).

59 Suworow, J. K.: Allgemeine Fischkunde. VEB Deutscher Verlag der Wissenschaften, Berlin 1959.

60 Vibert, R.: Possibilités et limites du repeuplement en truites et saumons par boîte d'alevinage. Bull. Off. Int. Cons. Sup. Pêche, No. 37, IV (1959).

61 Wagner, H.: Einiges vom Seesaibling. Carinthia II 57, 93-102 (1948).

62 Wanger, C.: Der Seesaibling. Schweiz. Fisch. Ztg., Sep. (1896).

63 Walter, E.: Die Fischerei als Nebenbetrieb des Landwirtes und Forstmannes. Neumann, Neudamm 1913.

64 Yamazaki, F.: Endocrinological studies on the reproduction of the female goldfish Carassius auratus $\mathrm{L}$. with special references to the function of the pituitary glands. Mem. Fac. Fish., Hokaido Univ. 13, 1-64 (1965).

65 Zeder, F.: Bericht über die Untersuchung des Zugersees und dessen Zuflüsse durch das kantonale Laboratorium Zug, polycopiert (1971-1974).

66 Züllig, H.: Sedimente als Ausdruck des Zustandes eines Gewässers. Schweiz. Z. Hydrol. 18, 5-144 (1956).

Tabelle 1. Morphometrische und hydrologische Daten des Zugersees.

Table 1. Morphometric and hydrological data of Lake Zug.

\begin{tabular}{ll} 
Meereshöhe & $413 \mathrm{~m}$ \\
Grösste Länge & $13,4 \mathrm{~km}$ \\
Grösste Breite & $4,6 \mathrm{~km}$ \\
Kleinste Breite & $0,8 \mathrm{~km}$ \\
Grösste Tiefe & $198 \mathrm{~m}$ \\
Mittlere Tiefe & $80 \mathrm{~m}$ \\
Seeoberfläche & $38,2 \mathrm{~km}^{2}$ \\
Seevolumen & $3,2 \mathrm{~km}^{3}$ \\
Einzugsgebiet & $246 \mathrm{~km}^{2}$ \\
Zufluss & $7,78 \mathrm{~m}^{3} / \mathrm{sec}$ \\
Zufluss Obersee & $2,96 \mathrm{~m}^{3} / \mathrm{sec}$ \\
Zufluss Untersee & $4,82 \mathrm{~m}^{3} / \mathrm{sec}$ \\
Aufenthaltszeit (theor.) & $13,0 \mathrm{Jahre}$ \\
Aufenthaltszeit: & \\
Obersee & $20,7 \mathrm{Jahre}$ \\
Untersee & $8,4 \mathrm{Jahre}$ \\
\hline
\end{tabular}


Tabelle 2. Transparenz des Zugersees um die Jahrhundertwende und in den letzten Jahren.

Table 2. Transparency of Lake Zug at the turn of the century and during the past few years.

\begin{tabular}{|c|c|c|c|c|c|c|c|c|c|c|c|c|}
\hline $\begin{array}{l}\text { Transpa } \\
\text { Monat } \\
\text { Jahr }\end{array}$ & $\begin{array}{c}\text { enz (in } \\
\text { Jan. }\end{array}$ & $\begin{array}{l}\text { m; Seccl } \\
\text { Feb. }\end{array}$ & $\begin{array}{l}\text { i-Scheib } \\
\text { März }\end{array}$ & e) & Mai & Juni & Juli & Aug. & Sep. & Okt. & Nov. & Dez. \\
\hline 1906 & & & & 12,0 & 3,8 & 5,5 & 4,3 & 4,4 & 6,2 & 8,4 & & \\
\hline 1907 & 11,8 & 16,0 & 11,8 & 8,9 & 11,8 & 6,2 & 4,6 & & 4,6 & 5,3 & & 10,9 \\
\hline 1908 & & 14,5 & 16,1 & 12,8 & 4,0 & 4,8 & 4,6 & 4,6 & 4,6 & 6,3 & 9,2 & 11,1 \\
\hline 1909 & 12,2 & 3,5 & 16,9 & 7,4 & & 5,5 & & & & & & \\
\hline 1970 & & 5,8 & & & 1,4 & & & & & & 12,6 & \\
\hline 1971 & & 6,2 & & & 1,6 & & 3,6 & & & & 7,0 & \\
\hline 1973 & & & & 2,0 & & 7,3 & 3,8 & & & & & \\
\hline
\end{tabular}

Tabelle 3. Färbung des Zugersees um die Jahrhundertwende und in den letzten Jahren (Skalenwerte nach Forel).

Table 3. Colour of Lake Zug at the turn of the century and during the past few years (readings after Forel).

\begin{tabular}{ll} 
Forel). & \\
\hline Jahr & Durchschnittlicher Skalenwert \\
\hline 1907 & $6-7$ \\
1908 & $5-9$ \\
1909 & $6-7$ \\
$1971-1973$ & $10-12$ \\
\hline
\end{tabular}

Tabelle 4. Vergleich einiger Grenzwerte für die Zuordnung zum eutrophen Seetyp und entsprechender Ist-Werte des Zugersees (Berechnungsgrundlage: Zeder [65], S. 12).

Table 4. Comparison of various critical values for the eutrophic categorization with corresponding current values of Lake Zug (calculation basis: Zeder [65], p. 12).

\begin{tabular}{|c|c|c|c|}
\hline & Sichttiefe & $\begin{array}{l}\text { Nährstoffgehalt } \\
\text { während Zirkulation }\end{array}$ & Nährstoffzufuhr \\
\hline $\begin{array}{l}\text { "Grenzwert } \\
\text { eutroph» }\end{array}$ & $3 \mathrm{~m}$ & $\begin{array}{c}30 \mu \mathrm{g} \mathrm{P} / 1 \\
500 \mu \mathrm{g} \mathrm{NO}-\mathrm{N} / 1\end{array}$ & 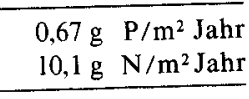 \\
\hline $\begin{array}{l}\text { Ist-Wert } \\
\text { Zugersee } \\
\end{array}$ & $\begin{array}{l}1,5 \mathrm{~m} \\
\text { (Mai 73) }\end{array}$ & $\begin{array}{c}100 \mu \mathrm{g} \mathrm{P} / 1 \\
2600 \mu \mathrm{g} \mathrm{NO}_{3}-\mathrm{N} / 1\end{array}$ & $\begin{array}{rr}10,0 \mathrm{~g} & \mathrm{P} / \mathrm{m}^{2} \mathrm{Jahr} \\
50,2 \mathrm{~g} \mathrm{~N} / \mathrm{m}^{2} \mathrm{Jahr} \\
\end{array}$ \\
\hline Referenz & Dobson & Vollenweider (1969) & Vollenweider (1969) \\
\hline
\end{tabular}

Tabelle 5. Häufigkeit der verschiedenen Futterorganismen in den Mageninhalten adulter, freilebender Seesaiblinge.

Table 5. Stomach contents of adult, free living arctic chars; frequency of the main food organisms.

\begin{tabular}{lc}
\hline Organismen & \% aller untersuchten Mageninhalte, in denen die \\
& Organismen gefunden wurden \\
\hline Planarien und Planarienkokons & 30 \\
Terr. Insekten & 5 \\
Chironomus & 30 \\
Nicht identifizierter Laich & 30 \\
Cyclops & 5 \\
Corethra & 50 \\
Daphnien & 25 \\
Bythotrephes & 5 \\
\hline
\end{tabular}


Tabelle 6. Kurvenwerte für die Längenwachstumskurven.

Table 6. Curve parameters of the age-length relationship.

\begin{tabular}{lllllr}
\hline Variable & $\begin{array}{l}\text { Kurvenwerte der } \\
\text { Wachstumskurve }\end{array}$ & Standardfehler & $\begin{array}{l}\text { Obere } \\
\text { Vertrauensgrenze }\end{array}$ & $\begin{array}{r}\text { Untere } \\
\text { Vertrauensgrenze }\end{array}$ \\
\hline $\mathrm{k}$ & $q$ & 0,191147 & 0,02526 & 0,191147 & 0,191147 \\
$\mathrm{t}_{0}$ & & 0,23914 & 0,08366 & 0,239138 & 0,239138 \\
$\mathrm{~L}_{x} \mathrm{~cm}$ & 49,64126 & 3,62086 & 49,64127 & 49,64125 \\
\hline $\mathrm{n}$ & & 223 & & & 0,21495 \\
\hline $\mathrm{k}$ & $\delta$ & 0,21495 & 0,03457 & 0,21495 & 0,331010 \\
$\mathrm{t}_{0}$ & 0,331010 & 0,068406 & 0,331010 & 47,290168 \\
$\mathrm{~L}_{x} \mathrm{~cm}$ & 47,290175 & 4,542636 & 47,290183 & \\
\hline $\mathrm{n}$ & 166 & & & & \\
\hline
\end{tabular}

Tabelle 7. Altersklassenzusammensetzung bei laichreifen Seesaiblingen.

Table 7 . Age class composition of spawing arctic chars.

\begin{tabular}{|c|c|c|c|c|c|c|c|c|c|}
\hline Alter & & $1^{+}$ & $2^{+}$ & $3^{+}$ & $4^{+}$ & $5^{+}$ & $6^{+}$ & $7^{+}$ & $8^{+}$ \\
\hline \multirow{6}{*}{$\begin{array}{l}\text { Ordentlicher } \\
\text { Laichfischfang } \\
\text { Netze mit } \\
30 \mathrm{~mm} \mathrm{MW}\end{array}$} & $\%$ & - & 7,1 & 59,5 & 28,6 & 4,8 & & & \multirow{6}{*}{0,4} \\
\hline & & & 3,2 & 35,5 & 8,7 & 3,4 & & & \\
\hline & $\%$ & - & 2,8 & 39,5 & 37,5 & 13,7 & 4,8 & 1,2 & \\
\hline & ${ }^{q} \sigma$ & - & 2,3 & 18,3 & 14,8 & 8,7 & 3,5 & 0,6 & \\
\hline & $d+q \%$ & - & 3,9 & 44,6 & 35,3 & 11,4 & 3,6 & 0,9 & \\
\hline & $\sigma$ & - & 0,8 & 24,6 & 20,0 & 7,4 & 3,9 & 0,4 & \\
\hline Kontrolle & ¿ \% & - & 30,7 & 61,5 & 7,8 & & & & \\
\hline Netze mit & $q \%$ & - & 13,2 & 66,0 & 6,6 & 13,2 & & & \\
\hline $\mathrm{MW} \leq 30 \mathrm{~mm}$ & $\delta+q \%$ & - & 21,4 & 64,3 & 7,1 & 7,2 & & & \\
\hline
\end{tabular}

Tabelle 8. Längen und Gewichte in verschieden warmem Wasser aufgezogener Seesaiblinge.

Table 8 . Lenghts and weights of arctic chars raised at various temperatures.

\begin{tabular}{|c|c|c|c|c|c|c|c|c|c|c|c|}
\hline \multirow[b]{2}{*}{ Argument } & \multicolumn{3}{|c|}{ Datum Kontrolle } & \multicolumn{2}{|l|}{$8^{\circ} \mathrm{C}$} & \multicolumn{2}{|l|}{$10^{\circ} \mathrm{C}$} & \multicolumn{2}{|c|}{$12^{\circ} \mathrm{C}$} & \multirow{2}{*}{$\begin{array}{l}14^{\circ} \mathrm{C} \\
\bar{x}\end{array}$} & \multirow[b]{2}{*}{$\sigma$} \\
\hline & & $\bar{x}$ & $\sigma$ & $\vec{x}$ & $\sigma$ & $\ddot{x}$ & $\sigma$ & $\bar{x}$ & $\sigma$ & & \\
\hline \multirow{4}{*}{$\begin{array}{l}\text { Längen } \\
\text { mm }\end{array}$} & 29.5 & 23,8 & 2,2 & 23,8 & 2,2 & 23,8 & 2,2 & 23,8 & 2,2 & 23,8 & 2,2 \\
\hline & 2.7 & 28,3 & 2,9 & 29,5 & 3,9 & 29,2 & 2,8 & 26,3 & 4,2 & 31,6 & 3,7 \\
\hline & 7.8. & 28,8 & 3,2 & 28,8 & 3,3 & 31,4 & 3,1 & 32,6 & 3,6 & 34,1 & 3,3 \\
\hline & 2.9. & 31,5 & 2,5 & 34,4 & 3,3 & 41,4 & 4,6 & 36,6 & 5,7 & 37,6 & 5,0 \\
\hline Gewichte & 29.5 & 15,5 & 8,3 & 15,5 & 8,3 & 15,5 & 8,3 & 15,5 & 8,3 & 15,5 & 8,3 \\
\hline \multirow{2}{*}{ tro., mg } & 7.8 & 23 & 11 & 24 & 11 & 32 & 18 & 34 & 15 & 44 & 18 \\
\hline & 2.9 & 33 & 14 & 44 & 18 & 97 & 44 & 59 & 34 & 71 & 40 \\
\hline
\end{tabular}


Tabelle 9. Produktionskosten für Seesaiblingsbesatzfische.

Table 9 . Production costs for arctic chars.

\begin{tabular}{|c|c|c|}
\hline Produktionskosten für & Fr. & Verfahren, Produktionserfolge, Bemerkungen \\
\hline \multirow[t]{5}{*}{1000 Brütlinge } & 37.93 & $\begin{array}{l}\text { Herkömmliches Streifverfahren, Bruterfolg } 60 \% \\
\text { Brutanstalt halb voll }\left(5 \times 10^{5} \text { Eier }\right)\end{array}$ \\
\hline & 29.93 & $\begin{array}{l}\text { Herkömmliches Streifverfahren, Bruterfolg } 60 \% \\
\text { Brutanstalt voll ( } 10^{6} \text { Eier) }\end{array}$ \\
\hline & 19.73 & Optimiertes Streifverfahren, Bruterfolg $90 \%$ \\
\hline & 14.40 & $\begin{array}{l}\text { Brutanstalt halb voll } \\
\text { Optimiertes Streifverfahren, Bruterfolg } 90 \%\end{array}$ \\
\hline & & Brutanstalt voll \\
\hline \multirow[t]{3}{*}{1000 Vorsömmerlinge } & 351.17 & $\begin{array}{l}\text { Bisheriges Aufzuchtverfahren, Aufzuchterfolge } 20 \% \\
\text { (Ist) }\end{array}$ \\
\hline & 74.32 & $\begin{array}{l}\text { Optimiertes Aufzuchtverfahren, minimal angestrebter } \\
\text { Aufzuchterfolg } 60 \% \text { (Soll) }\end{array}$ \\
\hline & 49.55 & Bei idealem Aufzuchterfolg von $90 \%$ \\
\hline 1000 Jährlinge & 525.50 & $\begin{array}{l}\text { Aufzuchterfolg } 85 \% \\
\text { (in den genannten Kosten sind die Produktionskosten } \\
\text { für das Ausgangsmaterial der nächst höheren Produk- } \\
\text { tionsstufe berücksichtigt) }\end{array}$ \\
\hline
\end{tabular}

Tabelle 10. Nutzen-Aufwand-Relationen für verschieden alt eingesetzte und nach verschiedenen Verfahren produzierte Seesaiblingsbesatzfische, $e=$ Effizienz.

Table 10. Cost-benefit relations for arctic chars of various age and produced by different methods.

\begin{tabular}{lll} 
Besatzfische & $\begin{array}{ll}\text { Produktions- } \\
\text { kosten }\end{array}$ & $\begin{array}{l}\text { Produktionskosten Rang } \\
\text { pro «geernteten» Fisch }\end{array}$ \\
\hline
\end{tabular}

Brütlinge

a) Herkömmliches Streifverfahren

- Brutanstalt voll

- Brutanstalt halb voll

b) Optimiertes Streifverfahren

- Brutanstalt voll

- Brutanstalt halb voll

$0,02993 / 0,0041=7.30 \quad 7$

$0,03793 / 0,0041=9.25$

$0,0144 / 0,0041=3.51 \quad 3$

$0,01973 / 0,0041=4.81 \quad 5$

Vorsömmerlinge

a) Bisheriges Aufzuchtverfahren

b) Optimiertes Aufzuchtverfahren

$0,35117 / 0,026=13.51 \quad 10$

$0,07432 / 0,026=2.86$

Jährlinge

a) Produktion mit Vorsömmerlingen aus herkömmlicher

Aufzucht

- Einsätze irgendwo

- Einsätze an idealen Einsatzorten (Untersee)

$0,93165 / 0,085=10.96$

9

b) Produktion mit Vorsömmerlingen aus optimierter

Aufzucht

- Einsätze irgendwo

$0,93165 / 0,205=4.54$

- Einsätze an idealen Einsatzorten (Untersee) 


\section{Ch. Ruhlé}

Tabelle 11. Berechnete relative Häufigkeiten der verschiedenen Altersklassen in den Fängen mit Netzen verschiedener Maschenweite; Fänge während der Laichzeit.

Table 11. Calculated relative frequency of the various age classes in the catches with nets of varying mesh size; catches during spawning season.

\begin{tabular}{|c|c|c|c|c|c|c|c|c|c|}
\hline $\begin{array}{l}\text { Altersklassen } \\
\text { (Laichzeit) }\end{array}$ & $\begin{array}{l}\text { Masch } \\
24\end{array}$ & $\begin{array}{l}\text { weiten } \mathrm{i} \\
26\end{array}$ & & 30 & 32 & 34 & 36 & 38 & 40 \\
\hline$\%$ & & & & & & & & & \\
\hline $2^{+}$ & 61,94 & $\begin{array}{l}55,79 \\
3107\end{array}$ & 49,77 & 35,37 & $\begin{array}{l}29,92 \\
40,61\end{array}$ & 19,20 & & $\begin{array}{r}9,01 \\
34,53\end{array}$ & $\begin{array}{r}2,74 \\
26.33\end{array}$ \\
\hline $3^{+}$ & $\begin{array}{r}28,32 \\
8,07\end{array}$ & $\begin{array}{l}31,07 \\
10,47\end{array}$ & 37,27 & $\begin{array}{l}38,42 \\
19,66\end{array}$ & $\begin{array}{l}40,61 \\
22,22\end{array}$ & $\begin{array}{l}43,62 \\
28,20\end{array}$ & $\begin{array}{l}39,47 \\
35,33\end{array}$ & 37,20 & 43,91 \\
\hline $\begin{array}{l}4^{+} \\
5^{+}\end{array}$ & $\begin{array}{l}8,07 \\
1,78\end{array}$ & $\begin{array}{r}10,47 \\
2,67\end{array}$ & $\begin{array}{r}11,01 \\
1,95\end{array}$ & $\begin{array}{r}19,66 \\
6,55\end{array}$ & $\begin{array}{r}22,22 \\
7,25 \\
\end{array}$ & $\begin{array}{r}28,20 \\
8,97\end{array}$ & 14,30 & 19,26 & 27,02 \\
\hline & $\simeq 100$ & $\simeq 100$ & $\simeq 100$ & $\simeq 100$ & $\simeq 100$ & $\simeq 100$ & $\simeq 100$ & $\simeq 100$ & $\simeq 100$ \\
\hline
\end{tabular}

Adresse des Autors: Dr. Ch. Ruhle, dipl. Forsting. ETH, Seenforschungslaboratorium der EAWAG/ETH, CH-6047 Kastanienbaum. 